\title{
Zur Bewertung von Niederdeutsch und lokalem Substandard in Hamburg
}

Lara Neumann/Ingrid Schröder (Hamburg)

\begin{abstract}
Based on the results of a quantitative study it is to be shown how Low German and forms of the Substandard of Hamburg are estimated within the population. The study was conducted within the context of the project Einstellungen gegenüber regionalen Sprachformen in der Großstadt: Niederdeutsch in Hamburg (NiH) (Attitudes towards regional languages in the city: Low German in Hamburg). The article takes a closer look at the connection between evaluation and social parameters such as age, language skills, origin and education. Finally, the potential differences in the evaluation between Low German and the vernacular Hamburg speech will be examined.
\end{abstract}

\section{Das Projekt: Gegenstand, Ziele, Design}

Obwohl Hamburg aufgrund seiner Sprach- und Kulturgeschichte durch die andauernde Koexistenz von Niederdeutsch und Hochdeutsch (cf. Bichel 1985: 1868; Möhn 2003: 2307) eine gewisse Sonderstellung einnimmt und dadurch als Forschungsobjekt unter regionalsprachlicher Perspektive besonders vielversprechend erscheint, hat das ortsspezifische Sprachspektrum im Bereich der Spracheinstellungsforschung bisher fast ausschließlich in den wenigen überregionalen quantitativen Studien - zumeist am Rande - Beachtung gefunden (Stellmacher 1987; Möller 2008; Möller 2010; Gärtig/Plewnia/Rothe 2010; Hundt 1992). In qualitativer Hinsicht ist das hamburgische Niederdeutsch eher in historisch ausgerichteten Analysen (Lesle 1986; Schröder 2007, 2012, i. E.) berücksichtigt worden und in Bezug auf die gegenwärtige Sprachsituation bis in die jüngste Zeit unerforscht geblieben. Ausnahmen bilden die rezenten Studien zur Salienz sprachlicher Merkmale (Hettler 2013; 2014), zu Sprachwahrnehmung und Zuschreibungspraxis (Schröder 2010; Schröder 2013; Jürgens 2015; Jürgens/Schröder 2016) und zum Enregisterment (Jürgens 2016).

Im Projekt Einstellungen gegenüber regionalen Sprachformen in der Großstadt: Niederdeutsch in Hamburg (NiH), das seit 2014 an der Universität Hamburg loziert ist, ${ }^{1}$ soll dieses Desiderat eingelöst werden. Es soll untersucht werden, welche Funktionen dem Dialekt und anderen regionalsprachlichen Formen im urbanen Raum gegenwärtig zukommen. Ausgangspunkt ist die Beobachtung, dass das Niederdeutsche in Hamburg trotz sinkender kommunika-

\footnotetext{
1 Wissenschaftliche Mitarbeiterinnen: Carolin Jürgens (bis August 2015), Lara Neumann (ab November 2015); studentische Hilfskräfte: Franziska Fleischhauer, André Pabst, Bernadette Schlaffner, Verena Turkowski. Ihnen ist die Aufbereitung der Daten zu danken.
}

Linguistik online 85, 6/17 - http://dx.doi.org/10.13092/lo.85.4088

CC by 3.0 
tiver Relevanz fest in Kultur und Medien verankert ist und öffentliche Beachtung findet. Das führt zu der Hypothese, dass das Niederdeutsche als besonderes, positiv bewertetes Ortsmerkmal wahrgenommen wird und die sozialsymbolische Funktion der Sprache gegenüber der kommunikativen Funktion an Bedeutung gewonnen hat. Im Zentrum des Projekts steht die Frage, welche Bewertungen und welches Identifikationspotential mit dem Niederdeutschen verbunden sind, d. h. inwieweit Niederdeutsch als Mittel der Identitätsstiftung und Identitätswahrung eingesetzt oder ggf. auch nur wahrgenommen wird. Im Vergleich und im Kontrast zum Niederdeutschen sollen auch die Einstellungen gegenüber dem lokalen Substandard in Hamburg untersucht werden. So ordnet sich das Projekt in den weiteren Rahmen der Wahrnehmungsdialektologie (cf. Hundt/Anders/Lasch 2010) und insbesondere in den Bereich der Spracheinstellungsforschung ein (cf. Cuonz 2014; grundlegend Lasagabaster 2004).

Das Projekt setzt sich aus zwei Teilstudien zusammen, eine qualitative und eine quantitative Teilstudie. In der qualitativen Teilstudie wurden 37 Interviews mit 39 Niederdeutschsprechern und Akteuren aus den Bereichen Kultur, Medien, offizielle Institutionen, Freizeiteinrichtungen und Politik durchgeführt. Ein besonderes Augenmerk lag dabei auf der Erfassung von Stereotypen, die als musterhafte Ausprägungen sowohl auf inhaltlicher wie auf formaler Ebene im Projektmaterial aufscheinen. Zur Untersuchung der Spracheinstellungen gegenüber dem Niederdeutschen und zusätzlich auch gegenüber dem hamburgischen Substandard wurden vor allem Daten zum Sprachwissen und zu Sprachbewertungen erhoben, um so die Zuschreibungen zu erfassen, die mit diesen Sprachformen verknüpft werden. Um der Hypothese einer spezifischen hamburgischen Identitätsstiftung nachzugehen, sind die Daten zu den Spracheinstellungen mit Daten zu Ortseinstellungen verbunden worden. Weitere Motivationen für den öffentlichen Gebrauch des Niederdeutschen sollten schließlich anhand der individuellen sprachbiographischen Erfahrungen der Interviewpartner ermittelt werden.

Die im Projektzusammenhang bisher durchgeführten qualitativen Auswertungen zeigen, dass dem Niederdeutschen in Hamburg tatsächlich eine besondere symbolische Funktion und damit ein hohes Identifikationspotential zukommt. Dabei spielen Stereotype eine Rolle, die eine Verbindung von Stadt und Sprache herstellen, z. B. Niederdeutsch als Sprache im Hafen/auf St. Pauli. Niederdeutsch wird zur Sprache der „echten Hamburger“" stilisiert (cf. Schröder 2010: 598; Schröder 2013: 380f.; Jürgens 2015: 259f.). In Bezug auf die lokalen Substandardformen scheinen solche Echtheits-Stereotype vor allem von jungen Leuten aufgerufen zu werden (cf. Schröder 2015: 49). Eingehendere Studien zur Verwendung und Bewertung des Substandards durch jüngere Sprecher fehlen bisher jedoch noch. ${ }^{2}$

In einer zweiten, quantitativen Teilstudie wurden die Einstellungen auch von solchen Bewohnern Hamburgs einbezogen, die sich nicht aktiv mit dem Niederdeutschen in kulturellen oder medialen Zusammenhängen auseinandersetzen. Im Rahmen einer standardisierten schriftlichen Befragung konnten 689 Fragebögen gesammelt werden. Davon sind 53 Fragebögen von den Teilnehmern der qualitativen Teilstudie ausgefüllt worden. Diese werden gesondert betrachtet, so dass Vergleiche zwischen den Antworten der Niederdeutsch-Akteure und der größeren Kontrollgruppe möglich sind. In der Auswertung wurden Sprachbewertungen, Sprach-

\footnotetext{
2 Diesem Themenkreis wird sich Lara Neumann in ihrem Dissertationsprojekt „Regionale Sprache als Identitätsmarker. Zur Konstruktion sozialer Bedeutung bei Hamburger Fußballfans“ widmen.
}

ISSN 1615-3014 
Zur Bewertung von Niederdeutsch und lokalem Substandard in Hamburg

verhalten und Sprachwissen hinsichtlich der in Hamburg verwendeten regionalen Sprachformen eingehend untersucht. Die sprachbezogenen Daten wurden mit den Daten zur eigenen sprachlichen Kompetenz und mit den Sozialdaten in Beziehung gesetzt. Im Folgenden sollen einige Ergebnisse dieser quantitativen Umfrage präsentiert werden. Die Fragebögen der Niederdeutsch-Akteure sind dabei nicht einbezogen, so dass die Auswertung auf Basis von 636 Fragebögen erfolgt.

Für die quantitative Teilstudie ist ein Fragebogen entworfen worden, der den Titel Hamburger Alltagssprache trägt, um die Aufmerksamkeit der Informanten nicht zu sehr auf das Niederdeutsche zu lenken. Er ist in sechs Bereiche gegliedert: (1) Fragen zur Stadt Hamburg, um die Assoziationen zu ermitteln, die mit der Stadt verbunden werden; (2) Fragen zum Spracherwerb und Sprachgebrauch sowie zur Fremdsprachenkompetenz; (3) Fragen zur sprachlichen Vielfalt in Hamburg; (4) Fragen zum hamburgischen Substandard (,typisch hamburgische Umgangssprache“); (5) Fragen zum Niederdeutschen („Plattdeutsch“); (6) Sozialdaten. Neben geschlossenen und offenen Fragen zum sprachlichen Wissen stehen semantische Differentiale und Skalen zur Erhebung der Bewertungen.

Die Fragebögen wurden einerseits in Papierform in öffentlichen Einrichtungen, wie z. B. Bibliotheken oder auch kleineren Geschäften, ausgelegt, von Projektmitarbeiterinnen und Studierenden an Nachbarn und Bekannte verteilt und zusätzlich in elektronischer Form im Internet zugänglich gemacht. Eine Hamburger Tageszeitung bewarb die Aktion außerdem in einem Artikel.

In diesem Beitrag soll erörtert werden, wie das Niederdeutsche und die hamburgischen Substandardformen bewertet werden. Dabei wird gezeigt, welcher Zusammenhang zwischen Sprachbewertungen und Sprachkompetenz besteht und inwiefern die Bewertungen mit sozialen Parametern wie Alter, Geschlecht, Herkunft und Bildung korrelieren. Am Ende kann so auch geprüft werden, ob Niederdeutsch und hamburgischer Substandard unterschiedlichen Bewertungen unterliegen. Die Auswertung erfolgte über Signifikanztests, die unter Berücksichtigung der vorliegenden Skalenniveaus ausgewählt wurden. Neben den Testergebnissen werden außerdem markante oder allgemein auffallende statistische Befunde anhand von Kreuztabellen dargestellt. Ausgehend von den vorliegenden Studien lassen sich folgende Hypothesen zur Bewertung des Niederdeutschen bilden:

(1) Eine bessere Niederdeutschkompetenz ist mit einer positiveren Bewertung verbunden. Bereits Stellmacher konnte dies aufgrund von Umfrageergebnissen aus dem Jahr 1984 eruieren: ,[D]ie positive Meinung zu einer Sprache wächst mit ihrer Beherrschung“ (Stellmacher 1987: 265). Bestätigt wird dieser Befund durch die Daten einer zweiten repräsentativen Umfrage aus dem Jahr 2007, in der die Eigenschaften ,humorvoll“ und „gemütlich“ dem Niederdeutschen häufiger von Befragten mit sehr guter/guter Kompetenz als von Befragten mit mäBiger bzw. geringer Kompetenz zugesprochen wurden (cf. Ipsos 2007).

(2) Da bei älteren Menschen eine höhere Dialektkompetenz festgestellt werden kann (cf. Stellmacher 1987: 27; Möller 2008: 66-67; Niebaum/Macha 2014: 208; cf. auch unten Kap. 4.1), ist zu erwarten, dass die Bewertungen mit steigendem Alter positiver werden. Zudem wird bei älteren Menschen eine höhere Bereitschaft sichtbar, sich für den Erhalt des Niederdeutschen einzusetzen (cf. Reershemius 2004: 92). Möller konnte altersspezifische ImageZuschreibungen erkennen, wobei die Gruppe der 50-Jährigen und Älteren Niederdeutsch stär- 
ker als „heimatlich“ und „humorvoll“ ansah, während die jüngste Befragtengruppe verhältnismäßig häufiger für „,nicht zeitgemäß“, „grob“ und „,nur für alte Menschen“ votierte (cf. Möller 2008: 65).

(3) Geschlechtsspezifische Unterschiede sind nicht zu prognostizieren. So konnte zwar in der bereits angeführten Umfrage von 1984 konstatiert werden, dass mehr Männer als Frauen Niederdeutsch sprechen und verstehen können, so dass auch bei Männern eine positivere Einstellung erwartbar ist (cf. Stellmacher 1987: 24-25), doch ist auf der anderen Seite die Hypothese formuliert worden, dass Frauen Dialekten aufgeschlossener gegenüberstehen als Männer (Stellmacher 1995: 25), ${ }^{3}$ die auf eine positivere Bewertung durch Frauen schließen lassen könnte.

(4) Eine Korrelation von Bildung und Bewertung des Niederdeutschen formuliert Möller auf Basis der Umfrage von 2007 zusammenfassend: „Den Zuschreibungen nach hat die Regionalsprache bei den Befragten mit Hauptschulabschluss eher ein Imageproblem als bei den Befragten höherer Bildungsabschlüsse“ (Möller 2008: 74). Dieses Ergebnis erstaunt angesichts der Tatsache, dass die Niederdeutschkompetenz bei Befragten mit Hauptschulabschluss stärker ausgeprägt ist als bei Befragten mit höheren Schulabschlüssen (cf. Möller 2008: 75; Stellmacher 1987: 29).

(5) Der angenommene Zusammenhang von Kompetenz und Bewertung lässt auch die Hypothese zu, dass Befragte, die im Sprachgebiet geboren und aufgewachsen sind, aufgrund ihrer potentiellen Kenntnis bzw. Vertrautheit mit der Sprache dieser positiver gegenüberstehen als Befragte, die in einem anderen Sprachraum aufgewachsen sind, so dass ein Zusammenhang zwischen Bewertung und Herkunftsregion zu erwarten ist (cf. auch Gärtig/Plewnia/Rothe 2010: 155).

(6) Inwiefern die Bewertungen für die hamburgische Umgangssprache andere Tendenzen zeigen als für das Niederdeutsche, lässt sich nur sehr vorsichtig abschätzen. Erste Beobachtungen führen zu dem Schluss, dass die hamburgische Umgangssprache von Jüngeren eine höhere Wertschätzung erfährt als von Älteren und ein verdecktes Prestige besitzt (cf. Schröder 2015: 49-51).

\section{Zur Bewertung des Niederdeutschen}

Die Bewertungen des Niederdeutschen und auch des hamburgischen Substandards sind mithilfe eines semantischen Differentials abgefragt worden. ${ }^{4}$ Dabei konnten für die beiden Werte

\footnotetext{
${ }^{3} \mathrm{Zu}$ den widersprüchlichen Positionen cf. auch Niebaum/Macha 2014: 205.

${ }^{4}$ Die Werte des semantischen Differentials, das zur Elizitierung der Bewertungen herangezogen wurde, werden in dieser Studie als abhängige Variablen untersucht. Hierfür wird ein Ordinalskalenniveau angenommen. Als unabhängige Variablen, die als potentielle Einflussfaktoren auf die Bewertungen angesehen werden, fungieren die Kompetenz, das Alter, das Geschlecht, die Herkunftsregion sowie das Bildungsniveau der Befragungsteilnehmer. Zu den verwendeten Tests zählt zum einen der Chi-Quadrat-Test auf Unabhängigkeit, der bei unabhängigen Variablen auf Nominalskalenniveau (Geschlecht) herangezogen wird. Der Testbericht enthält die Prüfstatistik Chi-Quadrat $\left(\chi^{2}\right)$, die Freiheitsgrade $(d f)$, den Signifikanzwert ( $p$ ) sowie die darauf basierende Effektstärke (Cramérs V). Darüber hinaus wird bei genau zwei Ausprägungen der unabhängigen Variable (Geschlecht) der Mann-Whitney-U-Test durchgeführt, der mit dem z-Wert sowie dem Signifikanzwert berichtet wird. Weist die unabhängige Variable mehr als zwei Ausprägungen auf (Kompetenz, Alter, Herkunftsregion, Bildungsniveau), wird eine Rangvarianzanalyse, der so genannte Kruskal-Wallis-H-Test, ausgeführt. Hier werden neben dem $\chi^{2}-$
} 
Zur Bewertung von Niederdeutsch und lokalem Substandard in Hamburg

eines Paars (z.B. „humorvoll - ernst“") jeweils zwei Stufen angekreuzt oder eine neutrale Option gewählt werden. In Tabelle 1 sind die Ergebnisse zu Frage 26 „Welche der folgenden Eigenschaften verbinden Sie persönlich am ehesten mit dem Plattdeutschen?“ (Basis: gültige Antworten) dargestellt. Die Bewertungspaare sind analog zur Forschungsliteratur (cf. zusammenfassend Jürgens 2015: 75-103) und auch aufgrund erster Auswertungen der qualitativen Teilstudie und etlicher Pretests festgelegt worden. Angegeben sind die Prozentzahlen sowie in Klammern gesetzt die absoluten Häufigkeiten auf Basis der 636 einbezogenen Fragebögen. Dunkel hinterlegt wurde die häufigste Nennung, hell die zweithäufigste Nennung.

\begin{tabular}{|l|l|l|l|l|l|l|l|}
\hline & & $\mathbf{2}$ & $\mathbf{1}$ & $\mathbf{0}$ & $\mathbf{- 1}$ & $\mathbf{- 2}$ & \\
\hline a. & humorvoll & $\begin{array}{l}35,2 \\
(210)\end{array}$ & $\begin{array}{l}42,8 \\
(255)\end{array}$ & $\begin{array}{l}14,9 \\
(89)\end{array}$ & $5,9(35)$ & $1,2(7)$ & ernst \\
\hline b. & vornehm & $0,9(5)$ & $4,5(26)$ & $\begin{array}{l}50,3 \\
(291)\end{array}$ & $\begin{array}{l}35,8 \\
(207)\end{array}$ & $\begin{array}{l}8,6 \\
(50)\end{array}$ & grob \\
\hline c. & gemütlich & $\begin{array}{l}44,6 \\
(265)\end{array}$ & $\begin{array}{l}38,9 \\
(231)\end{array}$ & $\begin{array}{l}13,3 \\
(79)\end{array}$ & $2,5(15)$ & $0,7(4)$ & ungemütlich \\
\hline d. & vertraut & $\begin{array}{l}31,7 \\
(187)\end{array}$ & $\begin{array}{l}43,6 \\
(257)\end{array}$ & $\begin{array}{l}17,6 \\
(104)\end{array}$ & $6,4(38)$ & $0,7(4)$ & distanziert \\
\hline e. & freundlich & $\begin{array}{l}31,2 \\
(185)\end{array}$ & $\begin{array}{l}41,0 \\
(243)\end{array}$ & $\begin{array}{l}19,7 \\
(117)\end{array}$ & $7,4(44)$ & $0,7(4)$ & $\begin{array}{l}\text { unfreund- } \\
\text { lich }\end{array}$ \\
\hline f. & modern & $1,5(9)$ & $3,4(20)$ & $\begin{array}{l}29,4 \\
(171)\end{array}$ & $\begin{array}{l}40,6 \\
(236)\end{array}$ & $\begin{array}{l}25,0 \\
(145)\end{array}$ & altmodisch \\
\hline g. & sympathisch & $\begin{array}{l}45,3 \\
(268)\end{array}$ & $\begin{array}{l}39,4 \\
(233)\end{array}$ & $\begin{array}{l}11,7 \\
(69)\end{array}$ & $2,7(16)$ & $1,0(6)$ & $\begin{array}{l}\text { unsympa- } \\
\text { thisch }\end{array}$ \\
\hline h. & cool & $\begin{array}{l}11,0 \\
(63)\end{array}$ & $\begin{array}{l}16,9 \\
(97)\end{array}$ & $\begin{array}{l}54,5 \\
(312)\end{array}$ & $\begin{array}{l}10,6 \\
(61)\end{array}$ & $\begin{array}{l}7,0 \\
(40)\end{array}$ & uncool \\
\hline i. & $\begin{array}{l}\text { geradeher- } \\
\text { aus }\end{array}$ & $\begin{array}{l}43,1 \\
(255)\end{array}$ & $\begin{array}{l}31,8 \\
(188)\end{array}$ & $\begin{array}{l}16,8 \\
(99)\end{array}$ & $6,6(39)$ & $\begin{array}{l}1,7 \\
(10)\end{array}$ & umständlich \\
\hline
\end{tabular}

Tabelle 1: Frage 26: „Welche der folgenden Eigenschaften verbinden Sie persönlich am ehesten mit dem Plattdeutschen?““

Die Eigenschaften, die mit dem Niederdeutschen häufiger verbunden werden, sind mehr „humorvoll“ als „ernst“, vorwiegend weder „grob“ noch „,vornehm“ mit Tendenz zu „grob“, mehr ,gemütlich“ als „ungemütlich“, mehr ,vertraut“ als „distanziert“, mehr ,freundlich“ als „unfreundlich“, vorwiegend weder ,altmodisch“ noch „,modern“ mit Tendenz zu ,altmo-

\footnotetext{
Wert auch die Freiheitsgrade sowie der $p$-Wert angeführt. Fällt das Ergebnis der Rangvarianzanalyse signifikant aus, werden zusätzliche Post-Hoc-Paarvergleiche (Mann-Whitney-U-Tests) angestellt, wobei das Alphaniveau für die Post-Hoc-Tests nach der Bonferroni-Methode adjustiert wird. Bei unabhängigen Variablen auf Ordinalskalenniveau (Kompetenz, Alter, Herkunftsregion, Bildungsniveau) wird darüber hinaus der Rangkorrelationskoeffizient nach Spearman ermittelt. Angegeben werden der Korrelationskoeffizient $\left(r_{s}\right)$, die Freiheitsgrade und der Signifikanzwert. Im Hinblick auf den ermittelten Korrelationskoeffizienten werden Werte zwischen 0,0 und 0,2 als geringe, Werte zwischen 0,2 und 0,5 als mittlere, Werte zwischen 0,5 und 0,7 als hohe und Werte zwischen 0,7 und 1 als sehr hohe Korrelationen interpretiert (cf. Gries 2008: 144). Zusätzlich gibt das Vorzeichen Aufschluss über die Art der Korrelation, d. h., ob sie als gleich- oder gegensinnig einzustufen ist. Als signifikant wird ein $p$-Wert $\leq 0,05$ erachtet.
} 
disch“, mehr „sympathisch“ als „unsympathisch“, vorwiegend weder „,cool“ noch „uncool“ mit leichter Tendenz zu „,cool“, mehr ,,geradeheraus“ als „umständlich“.

Niederdeutsch wird folglich überwiegend mit positiven Gefühlswerten verbunden, die Nähe und Vertrautheit beinhalten. Dies entspricht dem gängigen Bild, das die Forschungsliteratur vermittelt. Niederdeutsch wird mit den Zuschreibungen ,gemütlich“, ,vertraut“ und „,sympathisch" als Nahsprache konzeptualisiert (so auch Jürgens 2015: 389). Weiterhin sind die neutralen Einschätzungen bei den Paaren ,vornehm - grob“, „,modern - altmodisch“ und „,cool uncool“" auffällig. Hier werden nicht so sehr emotive Zuschreibungen erfragt, sondern eher stilistische Zuordnungen, die zurückhaltend beurteilt werden.

Einige der Daten lassen sich mit der Erhebung des Instituts für niederdeutsche Sprache (INS) von 2007 vergleichen. Dabei ist allerdings Vorsicht geboten, da sowohl die Formulierung der Fragestellung als auch die Gruppierung der Befragten nach Sozialdaten voneinander abweichen. Zudem liegen die Daten der INS-Umfrage nur partiell in der Auswertung von Möller (2008) vor, können aber durch das Tabellenmaterial des Ipsos-Instituts ${ }^{5}$ ergänzt werden, das die Befragung durchgeführt und die Daten tabellarisch aufbereitet hat. ${ }^{6}$ In der Erhebung des INS wurde gefragt, welche Eigenschaften mit Plattdeutsch/Niederdeutsch assoziiert werden (Möller 2008: 24). In der Erhebungsliste befanden sich die Merkmale „humorvoll“, ,gemütlich“, „nicht zeitgemäß“ und „grob“, die sich mit den Ergebnissen der NiH-Umfrage 2014/2015 vergleichen lassen (Tabelle 2):

\begin{tabular}{|l|l|l|l|}
\hline Merkmal & $\begin{array}{l}\text { Umfrage INS ge- } \\
\text { samt } \\
\text { (Möller 2008: 24; } \\
\text { Ipsos 2007) } \\
\text { in \% (N = 775) }\end{array}$ & $\begin{array}{l}\text { Umfrage INS Ham- } \\
\text { burg (Ipsos 2007) } \\
\text { in \% (N= 52) }\end{array}$ & $\begin{array}{l}\text { Umfrage NiH } \\
\mathbf{( 2 0 1 4 / 1 5 )} \\
\text { in \% }\end{array}$ \\
\hline humorvoll & 84,9 & 83,1 & $78,0(\mathrm{~N}=596)$ \\
\hline gemütlich & 79,3 & 82,0 & $83,5(\mathrm{~N}=594)$ \\
\hline $\begin{array}{l}\text { nicht zeitgemäß } \\
\text { (NiH: altmodisch) }\end{array}$ & 30,0 & 39,1 & $65,6(\mathrm{~N}=581)$ \\
\hline grob & 27,3 & 18,0 & $44,4(\mathrm{~N}=579)$ \\
\hline
\end{tabular}

Tabelle 2: Vergleich INS 2007 - NiH 2014/15

Insgesamt zeigen sich dieselben Tendenzen in den hohen Werten für die Eigenschaften ,humorvoll“ und „gemütlich“. Deutliche Unterschiede werden für die Eigenschaften ,nicht zeitgemäß“/,,altmodisch“ und ,grob“ sichtbar, deren Werte in der NiH-Studie deutlich höher ausfallen. Sicherlich spielt hier das Umfragedesign eine Rolle. Während bei der INS-Umfrage die Liste der Eigenschaften vorgelesen wurde und jeweils mit ,ja“/,,nein“/,,ich weiß nicht“/,keine Angabe“ geantwortet werden sollte, waren die Teilnehmer der NiH-Umfrage aufgefordert

\footnotetext{
5 Im Folgenden Ipsos 2007.

${ }^{6}$ Für die Bereitstellung der Tabellen danken wir Dr. Reinhard Goltz, Institut für niederdeutsche Sprache, Bremen.
} 
Zur Bewertung von Niederdeutsch und lokalem Substandard in Hamburg

worden, für alle vorgegebenen Eigenschaften eine abgestufte Bewertung auf einer zweipoligen Skala mit Bewertungspaaren abzugeben.

Um die Faktoren zu ermitteln, die mit der Bewertung des Niederdeutschen korrelieren, ist zuerst der Zusammenhang von Kompetenz und Bewertung zu überprüfen, sodann der des Alters, der Bildung, des Geschlechts sowie der Herkunftsregion.

\section{Zur Korrelation von Kompetenz und Bewertung des Niederdeutschen}

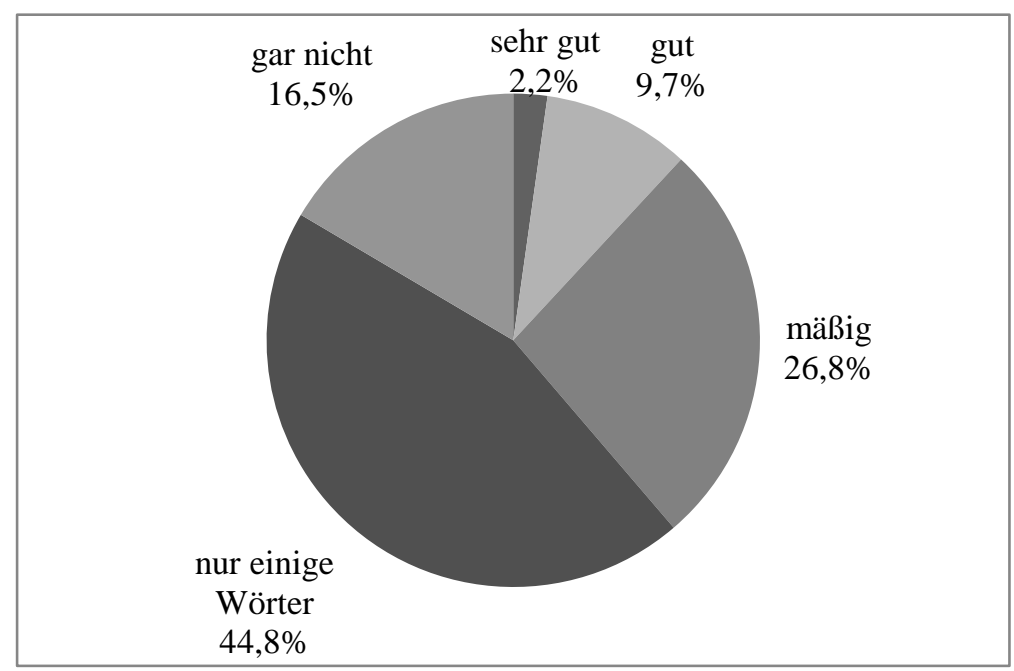

Diagramm 1: Frage 19: „Wie gut können Sie Plattdeutsch sprechen?““

Auf die Frage „Wie gut können Sie Plattdeutsch sprechen?“ (Frage 19) antworten 11,9\% aller Befragten ,sehr gut/gut“ (Basis: gültige Antworten; $\mathrm{N}=630$ ). 4,3\% geben an, dass Plattdeutsch ihre (erste oder zweite) Muttersprache ist (Frage 4; Basis: gültige Antworten; N = 623). 64,3\% behaupten von sich, dass sie Plattdeutsch ,sehr gut/gut“ verstehen (Frage 22; Basis: gültige Antworten; $\mathrm{N}=620$ ). In der INS-Umfrage haben dagegen 10\% der Hamburger Befragten auf die Kompetenzfrage geantwortet, dass sie ,sehr gut/gut“ Plattdeutsch sprechen (Möller 2010: 558); 46\% schätzten ihre passive Kompetenz als „,sehr gut/gut“ ein (Möller 2010: 556).

Im Hinblick auf den Zusammenhang von Kompetenz und Bewertung ist zu fragen, ob die Zuschreibung einer sehr guten/guten Kompetenz $\mathrm{zu}$ anderen Bewertungen führt als eine schlechtere oder gar fehlende aktive Sprachkompetenz (Tabelle 3).

\begin{tabular}{|l|l|l|}
\hline Bewertung & $\begin{array}{l}\text { sehr gut / gut } \\
\text { in \% }\end{array}$ & $\begin{array}{l}\text { gar nicht } \\
\text { in \% }\end{array}$ \\
\hline (eher) humorvoll ${ }^{7}$ & $100(13) / 90,8(49)$ & $63,5(61)$ \\
\hline (eher) gemütlich & $100(12) / 96,2(51)$ & $62,9(61)$ \\
\hline (eher) freundlich & $100(13) / 88,9(48)$ & $55,2(53)$ \\
\hline (eher) sympathisch & $100(12) / 94,5(51)$ & $64,6(62)$ \\
\hline
\end{tabular}

Tabelle 3: Korrelation von Kompetenz und Bewertung; Tendenz positiv

\footnotetext{
${ }^{7}$ Als „eher humorvoll/gemütlich“ usw. wird im Folgenden die Wahl der Stufe 1 paraphrasiert, als „humorvoll/gemütlich“" usw. das Votum für die Stufe 2.
} 
Zwischen den Bewertungspaaren „humorvoll - ernst“, „gemütlich - ungemütlich“, „,freundlich - unfreundlich“ und „sympathisch - unsympathisch“ sowie der Sprachkompetenz kann eine positive Korrelation festgestellt werden, d. h., dass mit steigender Kompetenz zunehmend die positiven Bewertungsoptionen gewählt werden. Der Zusammenhang ist in allen Fällen als mittelstark und hochsignifikant zu beschreiben, wobei der Korrelationskoeffizient für die Optionen ,gemütlich - ungemütlich“, „freundlich - unfreundlich“ und ,sympathisch unsympathisch“ etwas höher ausfällt als für das Eigenschaftspaar „humorvoll - ernst“. ${ }^{8}$ Die Differenzen in den Bewertungstendenzen werden auch im Rahmen des Kruskal-Wallis-HTests sichtbar. ${ }^{9}$ Dabei sind im direkten Gruppenvergleich vor allem Unterschiede in den Bewertungen zwischen den sehr guten bzw. guten Niederdeutschsprechern und den nichtkompetenten zu verzeichnen. Die Unterschiede sind für alle vier Eigenschaftspaare signifikant. ${ }^{10}$ Ein Blick in die prozentuale Verteilung zeigt, dass trotz der allgemein positiven Tendenz in allen Kompetenzstufen die weniger kompetenten Sprecher zurückhaltender urteilen und häufiger die Stufe 1 als die Stufe 2 für die jeweilige Eigenschaft wählen. Besonders deutlich sichtbar ist dies für das Merkmalspaar „freundlich - unfreundlich“: Während die Sprecher mit sehr guter bzw. guter Kompetenz zu 76,9\% (10) bzw. 57,4\% (31) die Stufe 2 „freundlich“ wählen und zu 23,1\% (3) bzw. 31,5\% (17) die Stufe 1 ,,eher freundlich“, sind es bei den nicht-kompetenten Sprechern nur 9,4\% (9) bei der Stufe 2 und 45,8\% (44) bei der Stufe 1.

\begin{tabular}{|l|l|l|}
\hline \multicolumn{1}{|c|}{ Kompetenz } & $\begin{array}{l}\text { sehr gut / gut } \\
\text { in \% }\end{array}$ & $\begin{array}{l}\text { gar nicht } \\
\text { in \% }\end{array}$ \\
\hline (eher) vertraut & $91,7(11) / 96,3(51)$ & $45,9(44)$ \\
\hline (eher) geradeheraus & $100(12) / 90,2(46)$ & $46,4(45)$ \\
\hline
\end{tabular}

Tabelle 4: Korrelation von Kompetenz und Bewertung; Tendenz positiv vs. Tendenz negativ

Die verschiedenen Kompetenzgruppen unterscheiden sich auch im Hinblick auf die Optionen „vertraut - distanziert“ und „geradeheraus - umständlich“ (Tabelle 4) signifikant in ihrer

\footnotetext{
8 Spearman-Korrelation (gemütlich/ungemütlich*Kompetenz): $r_{s}=0,349, d f=590, p_{\text {zweiseitig }}=0,000$; SpearmanKorrelation (freundlich/unfreundlich*Kompetenz): $r_{s}=0,341, d f=588, \quad p_{\text {zweiseitig }}=0,000$; Spearman-Korrelation (sympathisch/unsympathisch*Kompetenz): $r_{s}=0,332, d f=588, p_{\text {zweiseitig }}=0,000$; Spearman-Korrelation (humorvoll/ernst*Kompetenz): $r_{s}=0,264, d f=591, p_{\text {zweiseitig }}=0,000$.

9 Kruskal-Wallis-H-Test (humorvoll/ernst*Kompetenz): $\chi^{2}=41,948, d f=4, p_{\text {zweiseitig }}=0,000$; Kruskal-Wallis-HTest (gemütlich/ungemütlich*Kompetenz): $\chi^{2}=75,246, d f=4, p_{\text {zweiseitig}}=0,000$; Kruskal-Wallis-H-Test (freundlich/unfreundlich*Kompetenz): $\quad \chi^{2}=70,507, \quad d f=4, \quad p_{\text {zweiseitig }}=0,000 ; \quad$ Kruskal-Wallis-H-Test $\quad$ (sympathisch/unsympathisch*Kompetenz): $\chi^{2}=68,411, d f=4, p_{\text {zweiseitig }}=0,000$.

10 Mann-Whitney-U-Test (humorvoll/ernst*gar nicht/gut): $z=-5,183, p_{\text {zweiseitig }}=0,000$; Mann-Whitney-U-Test (humorvoll/ernst*gar nicht/sehr gut): $\quad z=-3,302, \quad p_{\text {zweiseitig }}=0,001$; Mann-Whitney-U-Test (gemüt-

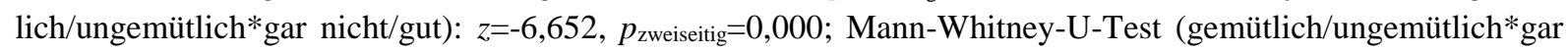
nicht/sehr gut): $z=-3,754, \quad p_{\text {zweiseitig }}=0,000 ;$ Mann-Whitney-U-Test (freundlich/unfreundlich*gar nicht/gut): $z=-6,161, p_{\text {zweiseitig }}=0,000$; Mann-Whitney-U-Test (freundlich/unfreundlich*gar nicht/sehr gut): $z=-4,803, p_{\text {zweisei- }}$ tig=0,000; Mann-Whitney-U-Test (sympathisch/unsympathisch*gar nicht/gut): $z=-6,325, p_{\text {zweiseitig }}=0,000$; MannWhitney-U-Test (sympathisch/unsympathisch*gar nicht/sehr gut): $z=-3,572, p_{\text {zweiseitig }}=0,000$.
} 
Zur Bewertung von Niederdeutsch und lokalem Substandard in Hamburg

zentralen Bewertungstendenz. ${ }^{11}$ Der vorliegende Zusammenhang ist für beide Gegensatzpaare positiv und mittelstark. ${ }^{12}$ Insbesondere zwischen den sehr guten bzw. guten und den nichtkompetenten Gewährspersonen ist ein vergleichsweise gegenläufiger Bewertungstrend festzustellen, ${ }^{13}$ der auch in der prozentualen Verteilung der Antworten deutlich wird: Während die kompetenten Sprecher zu über 90\% sagen, Niederdeutsch sei „(eher) vertraut“ bzw. „(eher) geradeheraus“, behaupten dies von den Befragten ohne Niederdeutschkompetenz weniger als die Hälfte. Dies könnte darauf hindeuten, dass sich in den Voten für die Kategorien ,vertraut“ und „umständlich“" weniger Bewertungen als die eigenen Kompetenzeinschätzungen widerspiegeln.

\begin{tabular}{|l|l|l|}
\hline \multicolumn{1}{|c|}{ Kompetenz } & $\begin{array}{l}\text { sehr gut / gut } \\
\text { in \% }\end{array}$ & $\begin{array}{l}\text { gar nicht } \\
\text { in \% }\end{array}$ \\
\hline Bewertung & $46,2(6) / 30,6(15)$ & $14,7(14)$ \\
\hline zwischen cool und uncool & $46,2(6) / 59,2(29)$ & $58,9(56)$ \\
\hline (eher) modern & $0,0(0) / 2,0(1)$ & $3,1(3)$ \\
\hline $\begin{array}{l}\text { zwischen modern und } \\
\text { altmodisch }\end{array}$ & $41,7(5) / 56,0(28)$ & $19,6(19)$ \\
\hline
\end{tabular}

Tabelle 5: Korrelation von Kompetenz und Bewertung; Tendenz positiv vs. Tendenz neutral

In Bezug auf die Bewertungspaare „cool - uncool“ und „modern - altmodisch“ wird von den verschiedenen Sprechergruppen überwiegend die neutrale Option gewählt (Tabelle 5). Dennoch kann eine schwache positive Korrelation zwischen den beiden Eigenschaftspaaren und der Niederdeutschkompetenz eruiert werden. ${ }^{14}$ Die Gruppe der kompetenten Sprecher folgt also auch in diesen Fällen dem allgemeinen Trend der leicht positiveren Eigenschaftszuschreibung. Die Differenzen in den Bewertungstendenzen sind allgemein statistisch auffällig. ${ }^{15}$ Dabei gehen besonders die Bewertungstrends der guten Sprecher und der nichtkompetenten Gewährspersonen auseinander. ${ }^{16}$ In Bezug auf das Merkmalspaar „,cool - uncool“ zeichnet sich in den kompetenten Sprechergruppen ein Trend zum positiven Pol ab, während beim Eigenschaftspaar „modern - altmodisch“ vor allem ein Trend zur neutralen Option zu erkennen ist.

\footnotetext{
${ }^{11}$ Kruskal-Wallis-H-Test (vertraut/distanziert*Kompetenz): $\chi^{2}=89,441, d f=4, p_{\text {zweiseitig }}=0,000 ;$ Kruskal-Wallis-HTest (geradeheraus/umständlich*Kompetenz): $\chi^{2}=82,951, d f=4, p_{\text {zweiseitig }}=0,000$.

12 Spearman-Korrelation (vertraut/distanziert*Kompetenz): $r_{s}=0,381, d f=586, \quad p_{\text {zweiseitig }}=0,000$; SpearmanKorrelation (geradeheraus/umständlich*Kompetenz): $r_{s}=0,369, d f=586, p_{\text {zweiseitig }}=0,000$.

13 Mann-Whitney-U-Test (vertraut/distanziert*gar nicht/gut): $z=-7,438, p_{\text {zweiseitig }}=0,000$; Mann-Whitney-U-Test (vertraut/distanziert*gar nicht/sehr gut): $z=-4,083, \quad p_{\text {zweiseitig }}=0,000 ;$ Mann-Whitney-U-Test (geradeheraus/umständlich*gar nicht/gut): $\quad z=-6,305, \quad p_{\text {zweiseitig }}=0,000 ; \quad$ Mann-Whitney-U-Test $\quad$ (geradeheraus/umständlich*gar nicht/sehr gut): $z=-4,424, p_{\text {zweiseitig }}=0,000$.

14 Spearman-Korrelation (cool/uncool*Kompetenz): $r_{s}=0,174, d f=570, p_{\text {zweiseitig }}=0,000$; Spearman-Korrelation (modern/altmodisch*Kompetenz): $r_{s}=0,145, d f=577, p_{\text {zweiseitig }}=0,000$.

15 Kruskal-Wallis-H-Test (cool/uncool*Kompetenz): $\chi^{2}=18,922, d f=4, p_{\text {zweiseitig }}=0,001$; Kruskal-Wallis-H-Test (modern/altmodisch*Kompetenz): $\chi^{2}=18,922, d f=4, p_{\text {zweiseitig }}=0,001$.

16 Mann-Whitney-U-Test (cool/uncool*gar nicht/gut): $z=-2,790, p_{\text {zweiseitig }}=0,005$; Mann-Whitney-U-Test (modern/altmodisch*gar nicht/gut): $z=-3,643, p_{\text {zweiseitig }}=0,000$.
} 
Die gängige Ansicht, dass Kompetenz und positive Bewertung des Niederdeutschen respektive des Dialekts miteinander verbunden sind (Stellmacher 1987: 15; cf. auch Gärtig/Plewnia/Rothe 2010: 155), kann bestätigt werden. In allen Fällen wird von den kompetenten Sprechern eine positivere Bewertung vorgenommen, auch wenn sich ein positiver Trend in vielen Fällen (,,humorvoll - ernst“, ,gemütlich - ungemütlich“, „freundlich - unfreundlich“, „sympathisch - unsympathisch“) bei allen Sprechergruppen zeigt. Bei den anderen Bewertungspaaren (,vertraut - distanziert“, „geradeheraus - umständlich“, „,cool - uncool“, „modern - altmodisch“) kann ferner eine Polarisierung mit positiverer bzw. neutralerer Einschätzung durch die kompetenten Sprecher und negativerer Einschätzung durch die nichtkompetenten Befragten bemerkt werden.

Auch die INS-Umfrage zeigte die beschriebenen Trends für die Eigenschaften ,gemütlich“ (sehr gute/gute Kompetenz: 95,4\%; keine Kompetenz: 66,6\%) und „humorvoll“ (sehr gute/gute Kompetenz: 93,1\%; keine Kompetenz: 74,9\%) (Ipsos 2007). Unterschiede bestehen hinsichtlich der Beurteilung der Eigenschaft ,nicht zeitgemäß“/,altmodisch“, wofür in Hamburg 77,3\% der nicht-kompetenten Sprecher votieren, in der INS-Umfrage aber nur 37,2\% dieser Sprechergruppe.

\section{$4 \quad$ Zur Korrelation von Sozialdaten und Bewertung des Niederdeutschen}

\subsection{Alter}

Im Hinblick auf mögliche Korrelationen von Sozialdaten und Bewertungen soll zuerst der potentielle Einfluss des Alters betrachtet werden. Die Befragten $(\mathrm{N}=615)$ sind in vier Gruppen unterteilt worden: 25 Jahre und jünger (12,2\% (75)), 26 bis 45 Jahre (32,0\% (197)), 46 bis 65 Jahre (32,8\% (202)), 66 Jahre und älter (22,9\% (141)). Fokussiert werden im Folgenden die Daten, die vom Gesamtbild (cf. oben, Abschnitt 3) abweichen.

Die Ergebnisse der ältesten Befragtengruppe ähneln stark denen der Gruppe der kompetenten Sprecher, die Ergebnisse der jüngsten Gruppe denen der nicht-kompetenten Sprecher, da zwischen diesen Gruppen große Überschneidungen bestehen bzw. sie miteinander korrelieren. ${ }^{17}$ So trauen sich 5,8\% (8) der Gruppe der 66-Jährigen und Älteren eine sehr gute und 18,1\% (25) eine gute Kompetenz zu. In der Gruppe der 46- bis 65-Jährigen sind es 0,5\% (1) bzw. 10,4\% (21) mit sehr guter bzw. guter Kompetenz. Ein anderes Bild zeigt sich bei den jüngeren Befragten: Von den 25-Jährigen und Jüngeren haben 2,7\% (2) eine sehr gute und 6,8\% (5) eine gute Kompetenz, bei der Gruppe der 26- bis 45-Jährigen sind es 1,0\% (2) bzw. 3,1\% (6). Die Niederdeutschkompetenz nimmt also von Generation zu Generation deutlich ab, bei leichter Stabilisierung in der jüngsten Generation. Auch wenn der Einfluss von Kompetenz und Alter nicht scharf getrennt werden kann, ist dennoch von Interesse, auf welche Weise sich die Sprachbewertungen der jüngeren Befragten von denen der älteren unterscheiden.

${ }^{17}$ Spearman-Korrelation (Kompetenz*Altersgruppen): $r_{s}=0,399, d f=607, p_{\text {zweiseitig }}=0,000$. 
Zur Bewertung von Niederdeutsch und lokalem Substandard in Hamburg

\begin{tabular}{|l|l|l|}
\hline \multicolumn{1}{|c|}{ Alter } & $\begin{array}{l}\text { 66 und älter } \\
\text { in \% }\end{array}$ & $\begin{array}{l}\mathbf{2 5} \text { und jünger } \\
\text { in \% }\end{array}$ \\
\hline (eher) humorvoll & $87,4(111)$ & $69,8(51)$ \\
\hline (eher) gemütlich & $93,5(115)$ & $68,1(49)$ \\
\hline (eher) vertraut & $85,0(102)$ & $64,4(47)$ \\
\hline (eher) freundlich & $90,4(113)$ & $53,4(39)$ \\
\hline (eher) sympathisch & $93,5(115)$ & $69,8(51)$ \\
\hline
\end{tabular}

Tabelle 6: Korrelation von Alter und Bewertung; Tendenz positiv

Viele Bewertungen weisen aufgefächert nach Altersgruppen dieselbe positive Tendenz auf (Tabelle 6). So votiert in allen Altersklassen die Mehrheit der Befragten für die positiv konnotierten Eigenschaften „(eher) humorvoll“, „(eher) gemütlich“, „(eher) vertraut“, „(eher) freundlich“ und „(eher) sympathisch“. Dennoch können signifikante Korrelationen zwischen den Altersgruppen und den Zuschreibungen konstatiert werden. Für die Eigenschaftspaare „gemütlich - ungemütlich“ und „freundlich - unfreundlich“ liegt der ermittelte Rangkorrelationskoeffizient etwas höher als für die Bewertungsoptionen „humorvoll - ernst““, „vertraut distanziert“ und „sympathisch - unsympathisch“. ${ }^{18}$ Der Anstieg des Alters geht in allen Fällen mit einer positiveren Bewertungstendenz einher. Ferner weisen auch die Ergebnisse der Rangvarianzanalyse Unterschiede zwischen den untersuchten Stichproben nach. ${ }^{19}$ Die paarweisen Gruppenvergleiche zeigen insbesondere zwischen der jüngsten und ältesten Altersgruppe hochsignifikante Unterschiede auf. ${ }^{20}$

\begin{tabular}{|l|l|l|}
\hline Bewertung & $\begin{array}{l}\text { 66 und älter } \\
\text { in \% }\end{array}$ & $\begin{array}{l}\mathbf{2 5} \text { und jünger } \\
\text { in \% }\end{array}$ \\
\hline (eher) uncool & $13,5(15)$ & $23,9(17)$ \\
\hline zwischen cool und uncool & $64,0(71)$ & $49,3(35)$ \\
\hline
\end{tabular}

Tabelle 7: Korrelation von Alter und Bewertung; Tendenz neutral

18 Spearman-Korrelation (gemütlich/ungemütlich*Altersgruppen): $r_{s}=0,321, d f=582, p_{\text {zweiseitig }}=0,000$; SpearmanKorrelation (freundlich/unfreundlich*Altersgruppen): $r_{s}=0,321, d f=583, p_{\text {zweiseitig }}=0,000$; Spearman-Korrelation (humorvoll/ernst*Altersgruppen): $\quad r_{s}=0,223, \quad d f=584, \quad p_{\text {zweiseitig }}=0,000 ; \quad$ Spearman-Korrelation (vertraut/distanziert*Altersgruppen): $\quad r_{s}=0,258, \quad d f=578, \quad p_{\text {zweiseitig }}=0,000 ; \quad$ Spearman-Korrelation $\quad$ (sympathisch/unsympathisch*Altersgruppen): $r_{s}=0,245, d f=581, p_{\text {zweiseitig }}=0,000$.

19 Kruskal-Wallis-H-Test (humorvoll/ernst*Altersgruppen): $\chi^{2}=32,936, d f=3, p_{\text {zweiseitig }}=0,000$; Kruskal-Wallis-HTest (gemütlich/ungemütlich*Altersgruppen): $\chi^{2}=61,153, d f=3, p_{\text {zweiseitig }}=0,000 ;$ Kruskal-Wallis-H-Test (vertraut/distanziert*Altersgruppen): $\quad \chi^{2}=38,925, \quad d f=3, \quad p_{\text {zweiseitig }}=0,000 ; \quad$ Kruskal-Wallis-H-Test $\quad$ (freundlich/unfreundlich*Altersgruppen): $\quad \chi^{2}=61,317, \quad d f=3, \quad p_{\text {zweiseitig }}=0,000 ; \quad$ Kruskal-Wallis-H-Test $\quad$ (sympathisch/unsympathisch*Altersgruppen): $\chi^{2}=35,621, d f=3, p_{\text {zweiseitig }}=0,000$.

20 Mann-Whitney-U-Test (humorvoll/ernst*25 und jünger/66 und älter): $z=-4,259, p_{\text {zweiseitig }}=0,000$; MannWhitney-U-Test (gemütlich/ungemütlich*25 und jünger/66 und älter): $z=-6,945, p_{\text {zweiseitig }}=0,000$; Mann-WhitneyU-Test (vertraut/distanziert*25 und jünger/66 und älter): $z=-4,853, p_{\text {zweiseitig }}=0,000 ;$ Mann-Whitney-U-Test (freundlich/unfreundlich*25 und jünger/66 und älter): $z=-6,923, p_{\text {zweiseitig }=0,000 ;}$ Mann-Whitney-U-Test (sympathisch/unsympathisch $* 25$ und jünger/66 und älter): $z=-5,14, p_{\text {zweiseitig }}=0,000$. 
Für das Wertepaar „,cool - uncool“ (Tabelle 7) kann dagegen keine Korrelation mit dem Alter nachgewiesen werden. ${ }^{21}$ Trotz Differenzen in der prozentualen Verteilung der Antworten zwischen der ältesten Generation und der jüngsten Gruppe können statistisch keine Unterschiede in der Bewertungstendenz eruiert werden. ${ }^{22}$

\begin{tabular}{|l|l|l|}
\hline \multicolumn{1}{|c|}{ Alter } & $\begin{array}{l}\mathbf{6 6} \text { und älter } \\
\text { in \% }\end{array}$ & $\begin{array}{l}\mathbf{2 5} \text { und jünger } \\
\text { in \% }\end{array}$ \\
\hline Bewertung & $30,7(35)$ & $60,2(44)$ \\
\hline $\begin{array}{l}\text { zwischen vornehm und } \\
\text { grob }\end{array}$ & $65,8(75)$ & $34,2(25)$ \\
\hline (eher) altmodisch & $50,4(58)$ & $80,8(59)$ \\
\hline $\begin{array}{l}\text { zwischen modern und } \\
\text { altmodisch }\end{array}$ & $46,1(53)$ & $12,3(9)$ \\
\hline
\end{tabular}

Tabelle 8: Korrelation von Alter und Bewertung; Tendenz negativ vs. Tendenz neutral

Bei den Eigenschaftspaaren „vornehm - grob“ und „modern - altmodisch“ überwiegen im Allgemeinen die eher negativ konnotierten bzw. neutralen Optionen (Tabelle 8). Dabei neigt die jüngste Generation stärker zu den Optionen ,(eher) grob“ und ,(eher) altmodisch“; die älteste Generation wählt dagegen vergleichsweise häufiger die neutralen Positionen. Der Zusammenhang ist also auch in diesem Fall gleichsinnig, wenngleich der Rangkorrelationskoeffizient für die Merkmalspaare „,vornehm - grob“ und ,modern - altmodisch“ schwächer ausfällt als bei den bisher dargestellten. ${ }^{23}$ Die unterschiedlichen Bewertungstrends, die der Kruskal-Wallis-H-Test für beide Wertepaare im Hinblick auf das Alter nachweist, ${ }^{24}$ sind vor allem auf die beschriebenen differenten Tendenzen der jüngsten und ältesten Generation zurückzuführen. ${ }^{25}$

\begin{tabular}{|l|l|l|}
\hline \multicolumn{1}{|c|}{ Alter } & $\begin{array}{l}\mathbf{6 6} \text { und älter } \\
\text { in \% }\end{array}$ & $\begin{array}{l}\mathbf{2 5} \text { und jünger } \\
\text { in \% }\end{array}$ \\
\hline Bewertung & $88,4(107)$ & $50,7(37)$ \\
\hline $\begin{array}{l}\text { (eher) geradeheraus } \\
\text { und umständlich }\end{array}$ & $9,9(12)$ & $32,9(24)$ \\
\hline
\end{tabular}

Tabelle 9: Korrelation von Alter und Bewertung; Tendenz positiv vs. Tendenz neutral

Des Weiteren hängt auch die Eigenschaftszuschreibung „geradeheraus - umständlich“ mit dem Alter der befragten Gewährspersonen zusammen (Tabelle 9). Der Spearman-

\footnotetext{
21 Spearman-Korrelation (cool/uncool*Altersgruppen): $r_{s}=0,003, d f=561, p_{\text {zweiseitig }}=0,923$.

22 Kruskal-Wallis-H-Test (cool/uncool*Altersgruppen): $\chi^{2}=0,995, d f=3, p_{\text {zweiseitig }}=0,802$.

23 Spearman-Korrelation (vornehm/grob*Altersgruppen): $r_{s}=0,148, \quad d f=569, \quad p_{\text {zweiseitig }}=0,000 ;$ SpearmanKorrelation (modern/altmodisch*Altersgruppen): $r_{s}=0,148, d f=570, p_{\text {zweiseitig }}=0,000$.

24 Kruskal-Wallis-H-Test (vornehm/grob*Altersgruppen): $\chi^{2}=14,955, d f=3, p_{\text {zweiseitig }}=0,002$; Kruskal-Wallis-HTest (modern/altmodisch*Altersgruppen): $\chi^{2}=13,212, d f=3, p_{\text {zweiseitig }}=0,004$.

25 Mann-Whitney-U-Test (vornehm/grob*25 und jünger/66 und älter): $z=-3,656, p_{\text {zweiseitig }}=0,000$; MannWhitney-U-Test (modern/altmodisch*25 und jünger/66 und älter): $z=-3,251, p_{\text {zweiseitig }}=0,001$.
} 
Zur Bewertung von Niederdeutsch und lokalem Substandard in Hamburg

Korrelationskoeffizient zeigt, dass die beiden Variablen in einem mittelstarken positiven $\mathrm{Zu}-$ sammenhang stehen. ${ }^{26}$ Der festgestellte Unterschied zwischen den untersuchten Stichproben manifestiert sich besonders im Einzelvergleich der ältesten und jüngsten Befragungsteilnehmer. ${ }^{27}$ Hier wählt die jüngste Altersgruppe mit 32,9\% vergleichsweise häufiger die neutrale Position; die älteste Generation votiert überwiegend und mit deutlichem Abstand zur jüngsten Generation für „(eher) geradeheraus“.

Insgesamt lassen sich die Bewertungen durch einen mehr oder weniger starken gleichläufigen Zusammenhang zum Alter charakterisieren. So sind die Prozentzahlen für die positiven Eigenschaften in den jüngeren Befragungsgruppen trotz allgemein positiver Bewertungstendenz niedriger als in den älteren Befragungsgruppen. Im Vergleich der beiden äußeren Altersgruppen fällt auf, dass die jüngste Generation das Niederdeutsche als mehr ,grob“ und mehr ,,altmodisch“ ansieht, während die älteste Gruppe hingegen meint, Niederdeutsch sei stärker ,geradeheraus".

Die Daten der INS-Umfrage (Möller 2008: 64; Ipsos 2007) weisen für „humorvoll“ und „gemütlich“ ebenfalls die höchsten Werte in der Gruppe der ältesten Befragten und die niedrigsten Werte in der Gruppe der jüngsten Befragten auf (für „humorvoll“: 50 Jahre und älter: 89,6\%, 35 bis 49 Jahre: 86,0\%, 34 Jahre und jünger: 75,5\%; für „gemütlich“: 50 Jahre und älter: $88,1 \%$, 35 bis 49 Jahre: $81,2 \%, 34$ Jahre und jünger: $61,9 \%$ ).

\begin{tabular}{|l|l|l|l|}
\hline \multicolumn{1}{|c|}{ Alter } & $\begin{array}{l}\text { 34 Jahre und jünger } \\
\text { INS 2007 } \\
\text { in \% }\end{array}$ & $\begin{array}{l}\text { 25 Jahre und jünger } \\
\text { NiH 2014/15 } \\
\text { in \% }\end{array}$ & $\begin{array}{l}\text { 26 bis 45 Jahre } \\
\text { NiH 2014/15 } \\
\text { in \% }\end{array}$ \\
\hline $\begin{array}{l}\text { nicht zeitge- } \\
\text { mäß/altmodisch }\end{array}$ & 38,3 & $80,8(59)$ & $70,9(136)$ \\
\hline grob & 36,5 & $60,2(44)$ & $48,5(93)$ \\
\hline
\end{tabular}

Tabelle 10: Korrelation von Alter und Bewertung; Vergleich INS - NiH

Unterschiede zwischen den Befragungen werden für die Eigenschaften „,nicht zeitgemäß“ und „grob“ deutlich (Tabelle 10). In der INS-Befragung (Ipsos 2007) liegen die Werte hierfür bei der jüngsten Befragtengruppe deutlich niedriger als in der NiH-Umfrage.

\subsection{Geschlecht}

Bei einer geschlechtsspezifischen Betrachtung zeigt sich, dass Männer (Anteil: 42,3\%) und Frauen (Anteil: 57,7\%) in der Regel zu gleichen Bewertungen tendieren $(\mathrm{N}=622)$. Dies gilt vor allem für die Bewertungspaare ,humorvoll - ernst“, „,vornehm - grob“, „modern - altmodisch“, „sympathisch - unsympathisch“, „,cool - uncool“ und ,geradeheraus - umständlich“, deren Testergebnisse (Chi-Quadrat-Test, Mann-Whitney-U-Test) allesamt nicht signifikant ausfielen. In diesen Fällen kann resümiert werden, dass beide Geschlechtergruppen den allgemeinen Tendenzen (cf. Tabelle 1) folgen.

\footnotetext{
26 Spearman-Korrelation (geradeheraus/umständlich*Altersgruppen): $r_{s}=0,333, d f=580, p_{\text {zweiseitig }}=0,000$.

27 Kruskal-Wallis-H-Test (geradeheraus/umständlich*Altersgruppen): $\chi^{2}=66,533, d f=3, p_{\text {zweiseitig }}=0,000$; MannWhitney-U-Test (geradeheraus/umständlich $* 25$ und jünger/66 und älter): $z=-7,067, p_{\text {zweiseitig }}=0,000$.
} 


\begin{tabular}{|l|l|l|}
\hline Bewertung & $\begin{array}{l}\text { meännlich } \\
\text { in \% }\end{array}$ & $\begin{array}{l}\text { weiblich } \\
\text { in \% }\end{array}$ \\
\hline (eher) gemütlich & $88,0(219)$ & $80,0(273)$ \\
\hline $\begin{array}{l}\text { zwischen gemütlich und } \\
\text { ungemütlich }\end{array}$ & $9,6(24)$ & $16,1(55)$ \\
\hline (eher) vertraut & $78,8(197)$ & $72,3(243)$ \\
\hline $\begin{array}{l}\text { zwischen vertraut und } \\
\text { distanziert }\end{array}$ & $15,2(38)$ & $19,6(66)$ \\
\hline (eher) freundlich & $72,9(183)$ & $71,3(241)$ \\
\hline $\begin{array}{l}\text { zwischen freundlich und } \\
\text { unfreundlich }\end{array}$ & $17,5(44)$ & $21,6(73)$ \\
\hline
\end{tabular}

Tabelle 11: Korrelation von Geschlecht und Bewertung; Tendenz positiv vs. Tendenz neutral

Der Chi-Quadrat-Unabhängigkeitstest und das darauf basierende Zusammenhangsmaß Cramérs V weisen für die Wertepaare ,gemütlich - ungemütlich“, „,vertraut - distanziert“ und „freundlich - unfreundlich“ (Tabelle 11) signifikante, wenn auch schwache Zusammenhänge nach. ${ }^{28}$ Bei diesen Eigenschaftspaaren tendieren Männer zu positiveren Bewertungen als Frauen, wobei die Frauen nicht stärker für die negativen Attribute optieren, sondern häufiger die neutrale Option wählen.

Die Ergebnisse zeigen insgesamt, dass geschlechtsspezifische Unterschiede gering ausfallen. Dass die Bewertungstendenzen bei Männern und Frauen gleich sind, belegen nicht zuletzt die nicht-signifikanten Ergebnisse des Mann-Whitney-U-Tests, der für alle neun Merkmalspaare durchgeführt wurde. Signifikante Unterschiede wie bei den Eigenschaftspaaren ,gemütlich ungemütlich“, „vertraut - distanziert“ und „,freundlich - unfreundlich“ ergeben sich vornehmlich dadurch, dass die neutrale Position in unterschiedlicher Frequenz gewählt wird. Ebenso wie in anderen Umfragen lassen sich im Hinblick auf geschlechtsspezifische Unterschiede folglich nur sehr vorsichtig Schlüsse ziehen. Vor allem die Hypothese, dass Frauen sprachlich aufgeschlossener sind als Männer (cf. Stellmacher 1995: 25), ist anhand der Einstellungsdaten nicht zu verifizieren. Vielmehr zeigen sich Frauen einer positiven Beurteilung gegenüber eher zurückhaltender als Männer.

\begin{tabular}{|l|l|l|l|l|}
\hline \multicolumn{1}{|r|}{ Geschlecht } & $\begin{array}{l}\text { m } \\
\text { INS 2007 } \\
\text { in \% }\end{array}$ & $\begin{array}{l}\text { w } \\
\text { INS 2007 } \\
\text { in \% }\end{array}$ & $\begin{array}{l}\text { m } \\
\text { NiH 2014 } \\
\text { in \% }\end{array}$ & $\begin{array}{l}\text { wiH 2014 } \\
\text { in \% }\end{array}$ \\
\hline humorvoll & 84,1 & 85,6 & $76,0(190)$ & $79,5(272)$ \\
\hline gemütlich & 79,2 & 79,5 & $88,0(219)$ & $80,0(273)$ \\
\hline grob & 29,5 & 25,1 & $44,5(110)$ & $44,0(145)$ \\
\hline $\begin{array}{l}\text { nicht zeitgemäß/ } \\
\text { altmodisch }\end{array}$ & 30,5 & 29,6 & $63,7(158)$ & $67,0(221)$ \\
\hline
\end{tabular}

Tabelle 12: Korrelation von Geschlecht und Bewertung; Vergleich INS - NiH

\footnotetext{
28 Chi-Quadrat-Test (gemütlich/ungemütlich*Geschlecht): $\chi^{2}=13,430, d f=4, p_{\text {zweiseitig }}=0,009$, Cramérs V=0,151; Chi-Quadrat-Test (vertraut/distanziert*Geschlecht): $\chi^{2}=9,646, d f=4, p_{\text {zweiseitig }}=0,047$, Cramérs V=0,128; ChiQuadrat-Test (freundlich/unfreundlich*Geschlecht): $\chi^{2}=13,662, d f=4, p_{\text {zweiseitig }}=0,008$, Cramérs V=0,152.
} 
Zur Bewertung von Niederdeutsch und lokalem Substandard in Hamburg

In der INS-Umfrage von 2007 (Möller 2008: 56; Ipsos 2007) waren im gesamten Sprachgebiet von den Frauen insgesamt leicht positivere Bewertungen vorgenommen worden, in der Projekt-Umfrage $(\mathrm{NiH})$ votieren Frauen hingegen deutlich weniger als Männer für die Eigenschaft „gemütlich“ und Frauen mehr als Männer für „altmodisch“, bei „grob“ im gleichen Maße (Tabelle 12).

\subsection{Herkunftsregion}

In Bezug auf die Herkunftsregion ${ }^{29}$ sind die Geburtsorte mit den Bewertungen korreliert worden $(\mathrm{N}=602)$. Dafür wurde die folgende Rangfolge gebildet: Geboren in der Region Hamburg (Anteil der Befragten: 56,0\% (337)), in Norddeutschland (24,3\% (146)), in Mittel- und Süddeutschland (13,8\% (83)), im Ausland (6,0\% (36)). Deutlich wird, dass die Distanz des Geburtsortes zu Hamburg bzw. zum niederdeutschen Sprachraum mit der Bewertung zusammenhängt, allerdings nicht bei allen Wertepaaren (Tabelle 13).

\begin{tabular}{|l|l|l|l|l|}
\hline \multicolumn{1}{|r|}{$\begin{array}{r}\text { Herkunfts- } \\
\text { region }\end{array}$} & $\begin{array}{l}\text { Nomburg } \\
\text { in \% }\end{array}$ & $\begin{array}{l}\text { Nord- } \\
\text { deutschland } \\
\text { in \% }\end{array}$ & $\begin{array}{l}\text { Mittel-/Süd- } \\
\text { deutschland } \\
\text { in \% }\end{array}$ & $\begin{array}{l}\text { Ausland } \\
\text { in \% }\end{array}$ \\
\hline $\begin{array}{l}\text { (eher) humor- } \\
\text { voll }\end{array}$ & $82,4(263)$ & $77,0(110)$ & $71,1(59)$ & $71,4(20)$ \\
\hline (eher) gemütlich & $87,4(278)$ & $84,5(120)$ & $74,3(61)$ & $58,6(17)$ \\
\hline (eher) vertraut & $81,2(255)$ & $78,4(113)$ & $53,1(43)$ & $57,2(16)$ \\
\hline $\begin{array}{l}\text { (eher) freund- } \\
\text { lich }\end{array}$ & $76,8(244)$ & $70,9(100)$ & $63,9(53)$ & $50,0(14)$ \\
\hline $\begin{array}{l}\text { (eher) sympa- } \\
\text { thisch }\end{array}$ & $87,0(276)$ & $85,4(122)$ & $75,6(62)$ & $75,9(22)$ \\
\hline $\begin{array}{l}\text { (eher) gerade- } \\
\text { heraus }\end{array}$ & $81,9(259)$ & $72,8(104)$ & $57,3(47)$ & $58,6(17)$ \\
\hline
\end{tabular}

Tabelle 13: Korrelation von Herkunftsregion und Bewertung; Tendenz positiv

Während die Korrelation von Herkunftsregion und Bewertung des Niederdeutschen für die Eigenschaftspaare „sympathisch - unsympathisch“, „,freundlich - unfreundlich“ und „,humorvoll - ernst“" gering ausfällt, kann für die Optionen „,gemütlich - ungemütlich“, „geradeheraus - umständlich“ und ,vertraut - distanziert“ ein mittlerer Zusammenhang vermerkt werden. ${ }^{30}$ Dabei nimmt mit zunehmender Distanz des Geburtsortes die positive Bewertung des Nieder-

\footnotetext{
${ }^{29}$ In Bezug auf die Variable „Herkunftsregion“ wurde nicht die primär räumliche Entfernung des Geburtsortes von Hamburg, sondern der sprachliche Abstand zugrunde gelegt, so dass Geburtsorte im nicht-niederdeutschen Sprachraum als entfernter eingestuft wurden als solche im niederdeutschen Sprachraum und Orte im nichtdeutschsprachigen Ausland entfernter als Orte im deutschen Sprachraum.

30 Spearman-Korrelation (sympathisch/unsympathisch*Herkunftsregion): $r_{s}=0,150, d f=569, \quad p_{\text {zweiseitig }}=0,000$; Spearman-Korrelation (freundlich/unfreundlich*Herkunftsregion): $r_{s}=0,153, d f=568, p_{\text {zweiseitg }}=0,000$; SpearmanKorrelation (humorvoll/ernst*Herkunftsregion): $r_{s}=0,180, d f=571, p_{\text {zweiseitig }}=0,000$; Spearman-Korrelation (gemütlich/ungemütlich*Herkunftsregion): $r_{s}=0,198, d f=569, p_{\text {zweiseitig }}=0,000$; Spearman-Korrelation (geradeheraus/umständlich*Herkunftsregion): $\quad r_{s}=0,212, \quad d f=568, \quad p_{\text {zweiseitig }}=0,000 ; \quad$ Spearman-Korrelation $\quad$ (vertraut/distanziert*Herkunftsregion): $r_{s}=0,229, d f=565, p_{\text {zweiseitig }}=0,000$.
} 
deutschen ab, die Korrelation kann damit als gleichsinnig bezeichnet werden. Ebenso weisen auch die signifikanten Befunde des Kruskal-Wallis-H-Tests auf Unterschiede in der Bewertungstendenz hin. ${ }^{31}$ Im direkten Gruppenvergleich sind insbesondere die signifikanten Unterschiede zwischen den im Ausland und in Hamburg Geborenen sowie zwischen den in Mittelbzw. Süddeutschland und den in Hamburg geborenen Gewährspersonen hervorzuheben. ${ }^{32}$ Die Bewertungstendenz der aus Norddeutschland stammenden Befragten unterscheidet sich lediglich für die Option ,geradeheraus - umständlich“ signifikant von den Eigenschaftszuschreibungen der gebürtigen Hamburger. ${ }^{33}$

\begin{tabular}{|l|l|l|l|l|}
\hline $\begin{array}{r}\text { region } \\
\text { regunfts- }\end{array}$ & $\begin{array}{l}\text { Hamburg } \\
\text { in \% }\end{array}$ & $\begin{array}{l}\text { Nord- } \\
\text { deutschland } \\
\text { in \% }\end{array}$ & $\begin{array}{l}\text { Mittel-/Süd- } \\
\text { deutschland } \\
\text { in \% }\end{array}$ & $\begin{array}{l}\text { Ausland } \\
\text { in \% }\end{array}$ \\
\hline $\begin{array}{l}\text { zwischen humor- } \\
\text { voll und ernst }\end{array}$ & $11,6(37)$ & $16,1(23)$ & $21,7(18)$ & $14,3(4)$ \\
\hline $\begin{array}{l}\text { zwischen gemüt- } \\
\text { lich und unge- } \\
\text { mütlich }\end{array}$ & $10,4(33)$ & $12,7(18)$ & $20,7(17)$ & $27,6(8)$ \\
\hline $\begin{array}{l}\text { zwischen vertraut } \\
\text { und distanziert }\end{array}$ & $14,6(46)$ & $15,3(22)$ & $28,4(23)$ & $25,0(7)$ \\
\hline $\begin{array}{l}\text { zwischen freund- } \\
\text { lich und un- } \\
\text { freundlich }\end{array}$ & $16,0(51)$ & $18,4(26)$ & $30,1(25)$ & $35,7(10)$ \\
\hline $\begin{array}{l}\text { zwischen gerade- } \\
\text { heraus und um- } \\
\text { ständlich }\end{array}$ & $13,0(41)$ & $17,5(25)$ & $25,6(21)$ & $31,0(9)$ \\
\hline
\end{tabular}

Tabelle 14: Korrelation von Herkunftsregion und Bewertung; Tendenz neutral

Die prozentuale Verteilung zeigt, dass die abnehmende positive Beurteilung, die mit zunehmender Distanz des Geburtsortes zu Hamburg eintritt, nicht zugleich mit einer Zunahme negativer Beurteilungen einhergeht (Tabelle 14): Bei den betrachteten Bewertungspaaren ist

\footnotetext{
31 Kruskal-Wallis-H-Test (humorvoll/ernst*Herkunftsregion): $\chi^{2}=19,403, d f=3, p_{\text {zweiseitig }}=0,000$; Kruskal-WallisH-Test (gemütlich/ungemütlich*Herkunftsregion): $\chi^{2}=23,710, d f=3, \quad p_{\text {zweiseitig }}=0,000 ; \quad$ Kruskal-Wallis-H-Test (vertraut/distanziert*Herkunftsregion): $\quad \chi^{2}=39,827, \quad d f=3, \quad p_{\text {zweiseitig }}=0,000 ; \quad$ Kruskal-Wallis-H-Test (freundlich/unfreundlich*Herkunftsregion): $\quad \chi^{2}=14,175, \quad d f=3, \quad p_{\text {zweiseitig }}=0,003 ; \quad$ Kruskal-Wallis-H-Test $\quad$ (sympathisch/unsympathisch*Herkunftsregion): $\chi^{2}=14,758, d f=3, p_{\text {zweiseitig }}=0,002$; Kruskal-Wallis-H-Test (geradeheraus/umständlich*Herkunftsregion): $\chi^{2}=27,731, d f=3, p_{\text {zweiseitig }}=0,000$.

32 Mann-Whitney-U-Test (humorvoll/ernst*Mittel- und Süddeutschland/Hamburg): $z=-3,979, p_{\text {zweiseitig }}=0,000$;

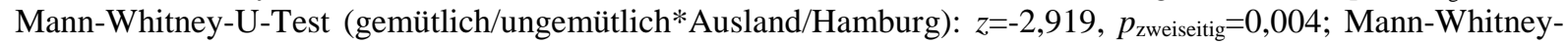
U-Test (gemütlich/ungemütlich*Mittel- und Süddeutschland/Hamburg): $z=-4,103, \quad p_{\text {zweiseitig }}=0,000$; MannWhitney-U-Test (vertraut/distanziert*Ausland/Hamburg): $\quad z=-3,132, \quad p_{\text {zweiseitig }}=0,002 ; \quad$ Mann-Whitney-U-Test (vertraut/distanziert*Mittel- und Süddeutschland/Hamburg): $z=-5,782, p_{\text {zweiseitig }}=0,000 ;$ Mann-Whitney-U-Test (freundlich/unfreundlich*Mittel- und Süddeutschland/Hamburg): $z=-3,137, p_{\text {zweiseitig }}=0,002$; Mann-Whitney-UTest (sympathisch/unsympathisch*Mittel- und Süddeutschland/Hamburg): $z=-3,659, p_{\text {zweiseitig }}=0,000$; MannWhitney-U-Test (geradeheraus/umständlich*Mittel- und Süddeutschland/Hamburg): $z=-4,889, p_{\text {zweiseitig }}=0,000$. 33 Mann-Whitney-U-Test (geradeheraus/umständlich*Norddeutschland/Hamburg): $z=-2,776, p_{\text {zweiseitig }}=0,006$.
} 
Zur Bewertung von Niederdeutsch und lokalem Substandard in Hamburg

vielmehr eine verstärkte Tendenz zur neutralen Position sichtbar, während die Werte für die negativen Bewertungen nur in geringem Umfang ansteigen.

\begin{tabular}{|l|l|l|l|l|}
\hline $\begin{array}{r}\text { Herkunfts- } \\
\text { region }\end{array}$ & $\begin{array}{l}\text { Hamburg } \\
\text { in \% }\end{array}$ & $\begin{array}{l}\text { Nord- } \\
\text { deutschland } \\
\text { in \% }\end{array}$ & $\begin{array}{l}\text { Mittel-/Süd- } \\
\text { deutschland } \\
\text { in \% }\end{array}$ & $\begin{array}{l}\text { Ausland } \\
\text { in \% }\end{array}$ \\
\hline (eher) ernst & $6,0(19)$ & $7,0(10)$ & $7,2(6)$ & $14,3(4)$ \\
\hline (eher) gemütlich & $87,4(278)$ & $84,5(120)$ & $74,3(61)$ & $58,6(17)$ \\
\hline $\begin{array}{l}\text { (eher) ungemüt- } \\
\text { lich }\end{array}$ & $2,2(7)$ & $2,8(4)$ & $4,9(4)$ & $13,8(4)$ \\
\hline $\begin{array}{l}\text { (eher) freund- } \\
\text { lich }\end{array}$ & $76,8(244)$ & $70,9(100)$ & $63,9(53)$ & $50,0(14)$ \\
\hline
\end{tabular}

Tabelle 15: Korrelation von Herkunftsregion und Bewertung; Tendenz negativ

Darüber hinaus werden gemäß den ermittelten positiven Korrelationen von Geburtsort und Sprachbewertungen von den im Ausland Geborenen verstärkt die Attribute „(eher) ernst“, „,(eher) ungemütlich“ und weniger ,,(eher) gemütlich“ und ,(eher) freundlich“ gewählt (Tabelle 15). Diese Beurteilungen stehen den gängigen Stereotypen gegenüber. Niederdeutsch wird von den Befragten weniger als Nahsprache konzeptualisiert.

Je weiter der Geburtsort von Hamburg entfernt ist, desto mehr weichen die Beurteilungen von denen der in Hamburg Geborenen ab. Dass die Anzahl der positiven Urteile mit steigender Entfernung des Geburtsortes sinkt, lässt vermuten, dass die Nähe des eigenen bzw. des aus dem Herkunftsort vertrauten Dialekts zum (hamburgischen) Niederdeutsch bzw. generell die Kenntnis des Niederdeutschen einen Einfluss auf die Bewertung ausübt. Dieser Befund passt zu den Daten zur kompetenzspezifischen Bewertung. Hier spiegelt sich der Zusammenhang von Kompetenz und auch Wissen bzw. Vertrautheit und der Bewertung einer Sprachform. Insofern ist es auch nicht weiter verwunderlich, dass für die im Ausland Geborenen gerade der Aspekt der Nahsprache eine geringere Bedeutung hat. Aber auch für diese Befragtengruppe hat Niederdeutsch überwiegend das Image einer „,humorvollen“, „gemütlichen“, „vertrauten“ und „sympathischen“ Sprache, die mehr ,geradeheraus“ als „umständlich“ ist.

\subsection{Bildung}

Bezüglich des Bildungsniveaus wurden die Abschlüsse von Hauptschule (5,7\% (35)), Realschule (19,7\% (121)), Gymnasium (73,0\% (448)), Fachschulen (1,0\% (6)) bzw. kein Abschluss $(0,7 \%$ (4)) unterschieden $(\mathrm{N}=614)$. Zur besseren Übersicht werden im Folgenden nur die Befragten mit und ohne Abitur (mit Abitur: 73,0\% (448); ohne Abitur: 27,0\% (166)) gegenübergestellt. ${ }^{34}$

\footnotetext{
34 Die statistische Auswertung bezieht dagegen alle differenzierten Skalenabstufungen des Bildungsabschlusses mit ein, da Korrelationen zwischen den untersuchten Variablen hier stärker hervortreten.
} 


\begin{tabular}{|l|l|l|}
\hline \multicolumn{1}{|c|}{ Bildung } & $\begin{array}{l}\text { ohne Abitur } \\
\text { in \% }\end{array}$ & $\begin{array}{l}\text { mit Abitur } \\
\text { in \% }\end{array}$ \\
\hline Bewertung & $80,8(122)$ & $73,6(315)$ \\
\hline (eher) vertraut sympathisch & $90,1(137)$ & $82,6(356)$ \\
\hline
\end{tabular}

Tabelle 16: Korrelation von Bildung und Bewertung; Tendenz positiv

Insgesamt wird deutlich, dass Niederdeutsch von Befragten ohne Abitur positiver bewertet wird als von Befragten mit Abitur (Tabelle 16). Bei einer Reihe von Bewertungspaaren kann eine negative Korrelation zum Schulabschluss festgehalten werden, d. h., dass mit ansteigendem Bildungsniveau die niederdeutsche Sprache negativer bewertet wird. So votieren die Befragten mit Abitur etwas zurückhaltender als die anderen, d. h. sie kreuzen häufiger die Stufe 1 an. Im Ganzen sind die ermittelten Zusammenhänge eher schwach, bspw. für die Eigenschaftspaare „vertraut - distanziert“ und „sympathisch - unsympathisch“. ${ }^{35}$ Der KruskalWallis-H-Test fällt zwar für diese beiden Merkmalspaare signifikant aus, ${ }^{36}$ nach Adjustierung des Alphaniveaus ist jedoch keiner der Post-Hoc-Einzelvergleiche statistisch auffällig.

\begin{tabular}{|l|l|l|}
\hline \multicolumn{1}{|c|}{ Bildung } & $\begin{array}{l}\text { ohne Abitur } \\
\text { in \% }\end{array}$ & $\begin{array}{l}\text { mit Abitur } \\
\text { in \% }\end{array}$ \\
\hline $\begin{array}{l}\text { (eher) gemüitlich - (eher) } \\
\text { ungemütlich }\end{array}$ & $88,9(137)-3,2(5)$ & $81,6(351)-3,2(14)$ \\
\hline (eher) cool - (eher) uncool & $32,6(47)-13,2(19)$ & $26,2(110)-19,3(81)$ \\
\hline
\end{tabular}

Tabelle 17: Korrelation von Bildung und Bewertung; Tendenz positiv vs. Tendenz negativ

Auch für die Bewertungspaare ,gemütlich - ungemütlich“ und „,cool - uncool“ kann nur eine schwache negative Korrelation nachgewiesen werden (Tabelle 17). ${ }^{37}$ Die Rangvarianzanalyse erzielt für das Merkmalspaar ,gemütlich - ungemütlich“ ein signifikantes Ergebnis; ${ }^{38}$ unterschiedliche Bewertungstendenzen können durch die nachfolgenden spezifischen Gruppenvergleiche aber nicht bestätigt werden. Vielmehr zeigt sich, dass mehrheitlich von beiden Gruppen die positive Option gewählt wird. Die Befragten mit Abitur entscheiden sich dennoch seltener als die Befragten ohne Abitur für die positive und dafür häufiger für die negative Option.

\footnotetext{
35 Spearman-Korrelation (vertraut/distanziert*Bildungsniveau): $r_{s}=-0,126, d f=577, p_{\text {zweiseitig }}=0,002$; SpearmanKorrelation (sympathisch/unsympathisch*Bildungsniveau): $r_{s}=-0,120, d f=581, p_{\text {zweiseitig }}=0,004$.

36 Kruskal-Wallis-H-Test (vertraut/distanziert*Bildungsniveau): $\chi^{2}=11,240, d f=4, p_{\text {zweiseitig }}=0,024$; KruskalWallis-H-Test (sympathisch/unsympathisch*Bildungsniveau): $\chi^{2}=11,302, d f=4, p_{\text {zweiseitig }}=0,023$.

37 Spearman-Korrelation (gemütlich/ungemütlich*Bildungsniveau): $r_{s}=-0,132, d f=582, p_{\text {zweiseitig }}=0,001$; Spearman-Korrelation (cool/uncool*Bildungsniveau): $r_{s}=-0,087, d f=562, p_{\text {zweiseitig }}=0,040$.

38 Kruskal-Wallis-H-Test (gemütlich/ungemütlich*Bildungsniveau): $\chi^{2}=15,838, d f=4, p_{\text {zweiseitig }}=0,003$.
} 
Zur Bewertung von Niederdeutsch und lokalem Substandard in Hamburg

\begin{tabular}{|l|l|l|}
\hline \multicolumn{1}{|c|}{ Bildung } & $\begin{array}{l}\text { ohne Abitur } \\
\text { in \% }\end{array}$ & $\begin{array}{l}\text { mit Abitur } \\
\text { in \% }\end{array}$ \\
\hline (eher) humorvoll & $82,2(129)$ & $76,4(328)$ \\
\hline $\begin{array}{l}\text { zwischen humorvoll und } \\
\text { ernst }\end{array}$ & $11,5(18)$ & $16,3(70)$ \\
\hline (eher) freundlich & $81,5(123)$ & $68,7(297)$ \\
\hline $\begin{array}{l}\text { zwischen freundlich und } \\
\text { unfreundlich }\end{array}$ & $11,3(17)$ & $23,1(100)$ \\
\hline (eher) geradeheraus & $82,4(126)$ & $72,4(311)$ \\
\hline $\begin{array}{l}\text { zwischen geradeheraus } \\
\text { und umständlich }\end{array}$ & $11,8(18)$ & $18,4(79)$ \\
\hline
\end{tabular}

Tabelle 18: Korrelation von Bildung und Bewertung; Tendenz positiv vs. Tendenz neutral

Weitere schwache gegensinnige Zusammenhänge sind zwischen dem Bildungsniveau und den Merkmalspaaren „,humorvoll - ernst“, „freundlich - unfreundlich“ und ,geradeheraus - umständlich“" auszumachen (Tabelle 18). ${ }^{39}$ Die Bewertungstendenzen für diese Optionen variieren sowohl im globalen Vergleich als auch für die spezifische Gegenüberstellung der Gewährspersonen mit Abitur gegenüber denen mit Hauptschul- oder Realschulabschluss. ${ }^{40}$ Dabei zeigen sich Tendenzen einer positiveren Bewertung durch die Befragten ohne Abitur; die Befragten mit Abitur wählen hingegen verstärkt die neutrale Option.

\begin{tabular}{|l|l|l|}
\hline \multicolumn{1}{|c|}{ Bildung } & $\begin{array}{l}\text { ohne Abitur } \\
\text { in \% }\end{array}$ & $\begin{array}{l}\text { mit Abitur } \\
\text { in \% }\end{array}$ \\
\hline Bewertung & $57,9(84)$ & $68,5(293)$ \\
\hline $\begin{array}{l}\text { zwer) altmodisch } \\
\text { altmodisch modern und }\end{array}$ & $35,9(52)$ & $27,1(116)$ \\
\hline
\end{tabular}

Tabelle 19: Korrelation von Bildung und Bewertung; Tendenz negativ

Bei dem Bewertungspaar „modern - altmodisch“ fällt auf, dass beide Befragtengruppen das Niederdeutsche mehrheitlich als „altmodisch“ einschätzen (Tabelle 19). Befragte mit Abitur wählen zwar frequenter die Option ,,(eher) altmodisch“, während sich Befragte ohne Abitur

39 Spearman-Korrelation (humorvoll/ernst*Bildungsniveau): $r_{s}=-0,178, d f=584, \quad p_{\text {zweiseitig }}=0,000$; SpearmanKorrelation (freundlich/unfreundlich*Bildungsniveau): $\quad r_{s}=-0,170, \quad d f=581, \quad p_{\text {zweiseitig }}=0,000 ; \quad$ SpearmanKorrelation (geradeheraus/umständlich*Bildungsniveau): $r_{s}=-0,151, d f=581, p_{\text {zweiseitig }}=0,000$.

40 Kruskal-Wallis-H-Test (humorvoll/ernst*Bildungsniveau): $\chi^{2}=21,143, d f=4, p_{\text {zweiseitig }}=0,000$; Mann-WhitneyU-Test (humorvoll/ernst*Haupt- und Volksschulabschluss/Abitur und höher): $z=-3,523, p_{\text {zweiseitig }}=0,000$; MannWhitney-U-Test (humorvoll/ernst*Realschulabschluss/Abitur und höher): $z=-3,122, p_{\text {zweiseitig }}=0,002$; KruskalWallis-H-Test (freundlich/unfreundlich*Bildungsniveau): $\chi^{2}=18,188, d f=4, p_{\text {zweiseitig }}=0,001$; Mann-Whitney-UTest (freundlich/unfreundlich*Haupt- und Volksschulabschluss/Abitur und höher): $z=-3,271, p_{\text {zweiseitig }}=0,001$; Mann-Whitney-U-Test (freundlich/unfreundlich*Realschulabschluss/Abitur und höher): $z=-3,058, \quad p_{\text {zweisei- }}$ tig $=0,002$; Kruskal-Wallis-H-Test (geradeheraus/umständlich*Bildungsniveau): $\chi^{2}=18,956, d f=4, p_{\text {zweiseitig }}=0,001$; Mann-Whitney-U-Test (geradeheraus/umständlich*Realschulabschluss/Abitur und höher): $z=-3,702, \quad p_{\text {zweisei- }}$ $\mathrm{tig}=0,000$. 
etwas häufiger für die neutrale Option entscheiden. Die prozentuale Diskrepanz zwischen den beiden Gruppen ist statistisch jedoch nicht von Bedeutung. ${ }^{41}$

\begin{tabular}{|l|l|l|l|l|}
\hline \multicolumn{1}{|c|}{ Bildung } & $\begin{array}{l}\text { ohne Abitur } \\
\text { INS 2007 } \\
\text { in \% }\end{array}$ & $\begin{array}{l}\text { mit Abitur } \\
\text { INS 2007 } \\
\text { in \% }\end{array}$ & $\begin{array}{l}\text { ohne Abitur } \\
\text { NiH 2014 } \\
\text { in \% }\end{array}$ & $\begin{array}{l}\text { mit Abitur } \\
\text { NiH 2014 } \\
\text { in \% }\end{array}$ \\
\hline humorvoll & 85,2 & 84,3 & $82,2(129)$ & $76,4(328)$ \\
\hline gemütlich & 78,8 & 79,6 & $88,9(137)$ & $81,6(351)$ \\
\hline grob & 31,1 & 21,6 & $39,5(56)$ & $46,7(200)$ \\
\hline $\begin{array}{l}\text { nicht zeitgemäß/ } \\
\text { altmodisch }\end{array}$ & 31,6 & 27,4 & $57,9(84)$ & $68,5(293)$ \\
\hline
\end{tabular}

Tabelle 20: Korrelation von Bildung und Bewertung; Vergleich INS - NiH

In der aktuellen Umfrage wurden bei den Befragten mit Abitur im Vergleich zur Gruppe ohne Abitur signifikant unterschiedliche Bewertungstendenzen für die Eigenschaftspaare „humorvoll - ernst“" und ,gemütlich - ungemütlich“ eruiert (Tabelle 20). Dabei stimmen die Gewährspersonen mit Abitur seltener für die Optionen „humorvoll“ und „gemütlich“. Umgekehrt können in dieser Stichprobe höhere Prozentzahlen für die Optionen „grob“ und ,altmodisch“ beobachtet werden.

Daher kann Möllers Feststellung, dass „die Regionalsprache bei den Befragten mit Hauptschulabschluss eher ein Imageproblem als bei den Befragten höherer Bildungsabschlüsse“ (Möller 2008: 74) habe, durch die vorliegende Umfrage nicht bestätigt werden. In der NiHUmfrage werden gegenläufige Tendenzen zur INS-Umfrage sichtbar.

Die Werte deuten auf einen Zusammenhang von alters- und bildungsspezifischer Bewertung hin, der auch statistisch durch eine mittelstarke negative Korrelation verifiziert werden kann. ${ }^{42}$ In den höheren Altersgruppen verfügen verhältnismäßig weniger Befragte über ein Abitur (66-Jährige und Ältere: 46,3\% (63); 46-65-Jährige: 69,0\% (138)) als in jüngeren Altersgruppen (26- bis 45-Jährige: 90,4\% (178); 25-Jährige und Jüngere: 88,0\% (66)). Der Einfluss von Bildung und Alter kann daher nicht getrennt werden.

\subsection{Zwischenergebnis: Faktoren für die Bewertung des Niederdeutschen}

Wenn nun noch einmal im Überblick gefragt wird, welche Faktoren mit den Eigenschaftszuschreibungen im Zusammenhang stehen, so fällt auf, dass Befragte mit einer höheren Kompetenz das Niederdeutsche positiver bewerten als Befragte mit einer niedrigen oder fehlenden Kompetenz. Mit zunehmendem Alter wird ebenfalls eine Tendenz zur positiveren Bewertung sichtbar. Ohne Frage spielt dabei eine Rolle, dass die älteren Befragten gleichzeitig eine höhere Sprachkompetenz aufweisen. Die geschlechtsspezifischen Unterschiede fallen hingegen gering aus. Dennoch wird sichtbar, dass insgesamt die Männer das Niederdeutsche leicht positiver einschätzen als die Frauen. Die Herkunftsregion spielt insbesondere dadurch eine Rolle, dass mit zunehmender Distanz des Geburtsortes von Hamburg die neutrale Position ver-

\footnotetext{
41 Spearman-Korrelation: (modern/altmodisch*Bildungsniveau): $r_{s}=-0,057, d f=571, p_{\text {zweiseitig }}=0,170$; KruskalWallis-H-Test (modern/altmodisch*Bildungsniveau): $\chi^{2}=3,103, d f=4, p_{\text {zweiseitig }}=0,541$.

42 Spearman-Korrelation (Altersgruppen*Bildungsniveau): $r_{s}=-0,360, d f=606, p_{\text {zweiseitig }}=0,000$.
} 
Zur Bewertung von Niederdeutsch und lokalem Substandard in Hamburg

stärkt gewählt wird und die positiven Bewertungen abnehmen. Für die im Ausland Geborenen wird das Niederdeutsche weniger als Nahsprache konzeptualisiert, worauf die vergleichsweise niedrigeren Werte für die Attribute „(eher) gemütlich“ und „(eher) freundlich“ hinweisen. Bei den Bildungsabschlüssen erweist sich, dass mit höherem Abschluss die negativen Bewertungen zunehmen. Als mögliche Einflussfaktoren treten am deutlichsten die Niederdeutschkompetenz, das Alter und die Herkunft hervor, weniger das Geschlecht und die Bildung.

\section{$5 \quad$ Niederdeutsch und hamburgischer Substandard im Vergleich}

Die Daten zum hamburgischen Substandard, im Fragebogen als „typisch hamburgische Umgangssprache“" abgefragt, sollen abschließend den Daten zum Niederdeutschen vergleichend gegenübergestellt werden. Die Tabelle enthält zusammengefasst die Werte für die Stufen 1 und 2, der Wert für die neutrale Position ist nicht angegeben (Tabelle 21).

\begin{tabular}{|l|l|l|l|l|l|}
\hline Eigenschaft & $\begin{array}{l}\text { ND } \\
\text { in \% }\end{array}$ & $\begin{array}{l}\text { Ham } \\
\text { in \% }\end{array}$ & $\begin{array}{l}\text { ND } \\
\text { in \% }\end{array}$ & $\begin{array}{l}\text { Ham } \\
\text { in \% }\end{array}$ & Eigenschaft \\
\hline humorvoll & $78,0(465)$ & $81,8(450)$ & $7,1(42)$ & $8,0(44)$ & ernst \\
\hline vornehm & $5,4(31)$ & $8,6(46)$ & $44,4(257)$ & $49,1(263)$ & grob \\
\hline gemütlich & $83,5(496)$ & $80,9(445)$ & $3,2(19)$ & $3,4(19)$ & ungemütlich \\
\hline vertraut & $75,3(444)$ & $71,4(391)$ & $7,1(42)$ & $9,9(54)$ & distanziert \\
\hline freundlich & $72,2(428)$ & $69,8(387)$ & $8,1(48)$ & $8,5(47)$ & unfreundlich \\
\hline modern & $4,9(29)$ & $11,9(64)$ & $65,6(381)$ & $40,0(216)$ & altmodisch \\
\hline sympathisch & $84,7(501)$ & $87,8(485)$ & $3,7(22)$ & $4,6(25)$ & unsympathisch \\
\hline cool & $27,9(160)$ & $35,2(190)$ & $17,6(101)$ & $13,1(71)$ & uncool \\
\hline geradeheraus & $74,9(443)$ & $85,9(474)$ & $8,3(49)$ & $2,3(13)$ & umständlich \\
\hline
\end{tabular}

Tabelle 21: Vergleich Niederdeutsch (ND) und hamburgische Umgangssprache (Ham)

Vergleicht man die Werte für das Niederdeutsche und für die hamburgische Umgangssprache, so fällt als Erstes ins Auge, dass beide Sprachformen grundsätzlich mit gleichen Tendenzen in der Werteverteilung bewertet werden. Für alle verglichenen Eigenschaftspaare können auffällige und hochsignifikante positive Korrelationen festgehalten werden. ${ }^{43}$ Obwohl die emotiven Eigenschaften „gemütlich“, „vertraut“, „freundlich“ für das Niederdeutsche leicht höhere Prozentwerte als für den hamburgischen Substandard aufweisen, ist der ermittelte Rangkorrelationskoeffizient für diese drei Merkmale am höchsten. Bei den Eigenschaften „humorvoll“ und ,sympathisch“ sind hingegen leicht höhere Werte für den Hamburger Substandard anzutreffen. Die beobachteten Unterschiede spiegeln sich auch im Korrelationskoeffizienten, wel-

43 Spearman-Korrelation (humorvoll/ernst (ND)*humorvoll/ernst (Ham)): $r_{s}=0,445, d f=530, p_{\text {zweiseitig }}=0,000$; Spearman-Korrelation (vornehm/grob (ND)*vornehm/grob (Ham)): $r_{s}=0,403, d f=516, p_{\text {zweiseitig }}=0,000$; Spearman-Korrelation (gemütlich/ungemütlich (ND)*gemütlich/ungemütlich (Ham)): $r_{s}=0,476, d f=528, p_{\text {zweisei- }}$ tig $=0,000$; Spearman-Korrelation (vertraut/distanziert (ND)*vertraut/distanziert (Ham)): $r_{s}=0,454, d f=526, p_{\text {zweisei- }}$ tig $=0,000$; Spearman-Korrelation (freundlich/unfreundlich (ND)*freundlich/unfreundlich (Ham)): $r_{s}=0,484$, $d f=530, p_{\text {zweiseitig }}=0,000$; Spearman-Korrelation (modern/altmodisch (ND)*modern/altmodisch (Ham)): $r_{s}=0,344$, $d f=520, p_{\text {zweiseitig }}=0,000$; Spearman-Korrelation (sympathisch/unsympathisch (ND)*sympathisch/unsympathisch (Ham)): $r_{s}=0,398, d f=529, p_{\text {zweiseitig }}=0,000 ;$ Spearman-Korrelation $(\mathrm{cool} /$ uncool (ND)*cool/uncool (Ham)): $r_{s}=0,441, \quad d f=512, \quad p_{\text {zweiseitig }}=0,000 ; \quad$ Spearman-Korrelation $\quad$ (geradeheraus/umständlich (ND)*geradeheraus/umständlich (Ham)): $r_{s}=0,434, d f=530, p_{\text {zweiseitig }}=0,000$. 
cher besonders für das Eigenschaftspaar „sympathisch - unsympathisch“ vergleichsweise niedrig ausfällt. Interessant sind darüber hinaus die Werte für die Paare „vornehm - grob“, „modern - altmodisch“ und „,cool - uncool“. Das Niederdeutsche erhält deutlich höhere Werte für „altmodisch“ und entsprechend weniger für „,modern“ als der Substandard. Zwar ist die Korrelation auch für dieses Eigenschaftspaar als mittelstark anzusehen, der Wert ist aber von allen verglichenen Paaren am niedrigsten. Bei dem Bewertungspaar „cool - uncool“ zeigt der Substandard die höheren Werte für „,Cool“ und geringere Werte für „uncool“. Ebenso ist es bei ,geradeheraus“ und „umständlich“. Deutlich höhere Werte sind für das Attribut ,grob“ für den hamburgischen Substandard ermittelt worden, zugleich aber auch leicht höhere Werte für „vornehm“; der Zusammenhang zwischen den Sprachbewertungen erweist sich dementsprechend als verhältnismäßig geringer. Dieses Ergebnis ist wahrscheinlich dem Umstand geschuldet, dass zwei Varianten des Substandards existieren, eine standardnahe prestigehafte, wie sie z. B. von Helmut Schmidt gesprochen wurde, und eine standardfernere mit eher geringerem Prestige oder ggf. auch verstecktem Prestige, wie es die Bezeichnungen „Barmbek Basch“ oder „Hamburger Kodderschnauze“ nahelegen, die von den Befragten genannt werden.

Die Untersuchung möglicher Einflussfaktoren auf die Bewertung zeigt Unterschiede hinsichtlich der Variablen „Gebrauch“,44 „Alter“ und „Herkunftsregion“.

\subsection{Zur Korrelation von Sprachgebrauch und Bewertung der hamburgischen Um- gangssprache}

\begin{tabular}{|l|l|l|}
\hline \multicolumn{1}{|c|}{ Sprachgebrauch } & $\begin{array}{l}\text { Hamburgisch } \\
\text { in \% }\end{array}$ & $\begin{array}{l}\text { nicht Hamburgisch } \\
\text { in \% }\end{array}$ \\
\hline (eher) humorvoll & $83,5(287)$ & $78,4(112)$ \\
\hline (eher) gemütlich & $84,5(289)$ & $73,8(107)$ \\
\hline (eher) vertraut & $78,6(267)$ & $55,5(80)$ \\
\hline (eher) freundlich & $73,5(253)$ & $60,7(88)$ \\
\hline (eher) sympathisch & $89,8(307)$ & $81,3(118)$ \\
\hline
\end{tabular}

Tabelle 22: Korrelation von Sprachgebrauch und Bewertung; Tendenz positiv

Obwohl in beiden Stichproben mehrheitlich für die positiven Eigenschaften votiert wird, kann der Mann-Whitney-U-Test für die Bewertungen der emotiven Eigenschaften „(eher) gemütlich“, „(eher) freundlich“ und „(eher) sympathisch“ (Tabelle 22) signifikante Unterschiede zwischen den Befragtengruppen nachweisen. ${ }^{45}$ Dabei schreiben die Befragten, die von sich selbst sagen, dass ihre Sprache durch hamburgische Merkmale gekennzeichnet ist, dem ham-

\footnotetext{
44 Aufgrund des unterschiedlichen Varietätenstatus und der damit verbundenen Wahrnehmung der beiden Sprachformen wurde für die hamburgische Umgangssprache nicht wie für das Niederdeutsche nach der aktiven und passiven Sprachbeherrschung gefragt, sondern ob jemand an der Aussprache als Hamburger/in erkannt wird. Daher wird im Folgenden „Sprachgebrauch“ anstelle von „Kompetenz“ als Kategorie verwendet.

45 Mann-Whitney-U-Test (gemütlich/ungemütlich*Sprachgebrauch): $z=-5,175, p_{\text {zweiseitig }}=0,000$; Mann-WhitneyU-Test (freundlich/unfreundlich*Sprachgebrauch): $z=-2,923, p_{\text {zweiseitig }}=0,003$; Mann-Whitney-U-Test (sympathisch/unsympathisch*Sprachgebrauch): $z=-4,215, p_{\text {zweiseitig }}=0,000$.
} 
Zur Bewertung von Niederdeutsch und lokalem Substandard in Hamburg

burgischen Substandard stärker positive emotive Eigenschaften zu als die Befragten, die meinen, ihre Sprache sei frei von hamburgischen Merkmalen. Dieser Zusammenhang kann für das Merkmalspaar „sympathisch - unsympathisch“ durch den Chi-Quadrat-Test bzw. den darauf basierenden Kontingenzkoeffizienten Cramérs V bestätigt werden. ${ }^{46}$ Ein noch größerer Unterschied ist für die Eigenschaft „(eher) vertraut“ zu konstatieren. ${ }^{47}$ Lediglich für die Option „(eher) humorvoll“ sind die Bewertungstendenzen beider Befragtengruppen so ähnlich, dass die beobachteten Unterschiede statistisch nicht ins Gewicht fallen. ${ }^{48}$ Insgesamt zeigen die Ergebnisse zum hamburgischen Substandard dieselben Tendenzen wie die für das Niederdeutsche. Dies betrifft sowohl die grundsätzlich positive Bewertung wie auch die Unterschiede bei den Kompetenz-/Gebrauchsgruppen und schließlich die große Differenz der Werte für „(eher) vertraut“. Wie in Bezug auf das Niederdeutsche könnten auch hier die Ergebnisse darauf hindeuten, dass das Merkmal ,vertraut“ nicht emotiv aufgefasst wird, sondern eher als eine Kategorie, die einen Wissensbestand beschreibt.

\subsection{Zur Korrelation von Alter und Bewertung der hamburgischen Umgangssprache}

\begin{tabular}{|l|l|l|}
\hline \multicolumn{1}{|c|}{ Alter } & $\begin{array}{l}\mathbf{6 6} \text { und älter } \\
\text { in \% }\end{array}$ & $\begin{array}{l}\mathbf{2 5} \text { und jünger } \\
\text { in \% }\end{array}$ \\
\hline (eher) humorvoll & $88,1(104)$ & $69,5(48)$ \\
\hline (eher) gemütlich & $92,3(109)$ & $72,5(50)$ \\
\hline (eher) vertraut & $79,3(92)$ & $58,0(40)$ \\
\hline (eher) freundlich & $87,7(107)$ & $60,8(42)$ \\
\hline (eher) sympathisch & $89,9(107)$ & $85,5(59)$ \\
\hline
\end{tabular}

Tabelle 23: Korrelation von Alter und Bewertung; Tendenz positiv

Unter Berücksichtigung aller Altersabstufungen ergeben sich für die Eigenschaftspaare ,humorvoll - ernst“, ,gemütlich - ungemütlich“, ,,vertraut - distanziert“, „,freundlich - unfreundlich“ und „sympathisch - unsympathisch“ (Tabelle 23) geringe bis mittlere positive Rangkorrelationen zwischen den Rängen der Variablen und den Altersgruppen. ${ }^{49}$ Der zusätzlich durchgeführte Kruskal-Wallis-H-Test bestätigt die abweichenden Bewertungstrends für alle Merkmalspaare bis auf „,sympathisch - unsympathisch“. ${ }^{50}$ Die prozentuale Verteilung für die

\footnotetext{
46 Chi-Quadrat-Test (sympathisch/unsympathisch*Sprachgebrauch): $\chi^{2}=20,056, d f=4, p_{\text {zweiseitig }}=0,000$, Cramérs $\mathrm{V}=0,203$.

47 Mann-Whitney-U-Test (vertraut/distanziert*Sprachgebrauch): $z=-5,175, p_{\text {zweiseitig }}=0,000$; Chi-Quadrat-Test (vertraut/distanziert*Sprachgebrauch): $\chi^{2}=32,320, d f=4, p_{\text {zweiseitig }}=0,000$, Cramérs V=0,258.

48 Chi-Quadrat-Test (humorvoll/ernst*Sprachgebrauch): $\chi^{2}=2,976, d f=4, p_{\text {zweiseitig }}=0,562$, Cramérs V=0,078; Mann-Whitney-U-Test (humorvoll/ernst*Sprachgebrauch): $z=-1,208, p_{\text {zweiseitig }}=0,227$.

49 Spearman-Korrelation (humorvoll/ernst*Altersgruppen): $r_{s}=0,199, \quad d f=539, \quad p_{\text {zweiseitig }}=0,000 ; \quad$ SpearmanKorrelation (gemütlich/ungemütlich*Altersgruppen): $r_{s}=0,210, d f=539, p_{\text {zweiseitig }}=0,000$; Spearman-Korrelation (vertraut/distanziert*Altersgruppen): $\quad r_{s}=0,183, \quad d f=536, \quad p_{\text {zweiseitig }}=0,000 ; \quad$ Spearman-Korrelation (freundlich/unfreundlich*Altersgruppen): $\quad r_{s}=0,288, \quad d f=544, \quad p_{\text {zweiseitig }}=0,000 ; \quad$ Spearman-Korrelation $\quad$ (sympathisch/unsympathisch*Altersgruppen): $r_{s}=0,087, d f=542, p_{\text {zweiseitig }}=0,042$.

50 Kruskal-Wallis-H-Test (humorvoll/ernst*Altersgruppen): $\chi^{2}=21,944, d f=3, p_{\text {zweiseitig }}=0,000$; Kruskal-Wallis-HTest (gemütlich/ungemütlich*Altersgruppen): $\chi^{2}=26,409, d f=3, p_{\text {zweiseitig }}=0,000$; Kruskal-Wallis-H-Test (vertraut/distanziert*Altersgruppen): $\quad \chi^{2}=19,9, \quad d f=3, \quad p_{\text {zweiseitig }}=0,000 ; \quad$ Kruskal-Wallis-H-Test $\quad$ (freund- 
emotiven Eigenschaften gleicht der, die auch für das Niederdeutsche sichtbar wurde: Die älteren Sprechergruppen nehmen positivere Bewertungen als die jüngsten Befragungsteilnehmer vor. Die anschließenden Post-Hoc-Einzelvergleiche können diese Beobachtungen validieren. Sie belegen u. a., dass zwischen der Gruppe der 66 Jahre und älteren Befragten und der jüngsten Gruppe (25 Jahre und jünger) ungleiche Bewertungstendenzen bestehen. ${ }^{51}$

\begin{tabular}{|c|c|c|c|c|c|c|}
\hline \multirow[t]{2}{*}{ Eigensch } & \multicolumn{2}{|c|}{ modern } & \multicolumn{2}{|c|}{$\begin{array}{l}\text { zwischen mo- } \\
\text { dern und altmo- } \\
\text { disch }\end{array}$} & \multicolumn{2}{|c|}{ altmodisch } \\
\hline & ND & Ham & ND & Ham & ND & Ham \\
\hline 66 und älter & $3,4(4)$ & $9,0(10)$ & $\begin{array}{l}46,1 \\
(53)\end{array}$ & $\begin{array}{l}47,3 \\
(53)\end{array}$ & $\begin{array}{l}50,4 \\
(58)\end{array}$ & $43,8(49)$ \\
\hline 25 und jünger & $6,9(5)$ & $\begin{array}{l}18,8 \\
(13)\end{array}$ & $12,3(9)$ & $\begin{array}{l}40,6 \\
(28)\end{array}$ & $\begin{array}{l}80,8 \\
(59) \\
\end{array}$ & $40,5(28)$ \\
\hline
\end{tabular}

\begin{tabular}{|c|c|c|c|c|c|c|}
\hline Eigenschaften & \multicolumn{2}{|l|}{ cool } & \multicolumn{2}{|c|}{$\begin{array}{l}\text { zwischen cool } \\
\text { und uncool }\end{array}$} & \multicolumn{2}{|c|}{ uncool } \\
\hline & ND & Ham & ND & Ham & ND & Ham \\
\hline 66 und älter & $\begin{array}{l}22,5 \\
(25)\end{array}$ & $\begin{array}{l}20,5 \\
(23)\end{array}$ & $\begin{array}{l}64,0 \\
(71)\end{array}$ & $\begin{array}{l}63,4 \\
(71)\end{array}$ & $\begin{array}{l}13,5 \\
(15)\end{array}$ & $16,1(18)$ \\
\hline 25 und jünger & $\begin{array}{l}26,8 \\
(19)\end{array}$ & $\begin{array}{l}52,2 \\
(36)\end{array}$ & $\begin{array}{l}49,3 \\
(35)\end{array}$ & $\begin{array}{l}39,1 \\
(27)\end{array}$ & $\begin{array}{l}23,9 \\
(17)\end{array}$ & $8,7(6)$ \\
\hline
\end{tabular}

Tabelle 24: Korrelation von Alter und Bewertung; Tendenz positiv vs. Tendenz negativ

Im Gegensatz zu den übrigen Eigenschaftspaaren zeichnet sich hinsichtlich der Merkmalspole „modern - altmodisch“ und „,cool - uncool“ (Tabelle 24) unter dem Faktor Alter eine gegenläufige Korrelation ab. ${ }^{52}$ Dementsprechend beurteilen die jüngeren Befragungsteilnehmer die Hamburger Umgangssprache sowohl stärker als „(eher) modern“ bzw. „(eher) cool“ als auch als weniger „(eher) altmodisch“ bzw. „(eher) uncool“. Deutlich werden die differenten Bewertungstendenzen besonders im Vergleich der voneinander entferntesten Altersgruppen, wobei sich die Zuschreibungstrends lediglich für das Merkmalspaar „cool - uncool“ als signifikant voneinander abweichend herausstellen. ${ }^{53}$ Dennoch besteht hier ein klarer Unterschied zur Beurteilung des Niederdeutschen, das bezüglich dieser Eigenschaften von der jüngeren

lich/unfreundlich*Altersgruppen): $\quad \chi^{2}=47,482, \quad d f=3, \quad p_{\text {zweiseitig }}=0,000 ; \quad$ Kruskal-Wallis-H-Test $\quad$ (sympathisch/unsympathisch*Altersgruppen): $\chi^{2}=5,049, d f=3, p_{\text {zweiseitig }}=0,168$.

51 Mann-Whitney-U-Test (humorvoll/ernst*25 und jünger/66 und älter): $z=-4,210, p_{\text {zweiseitig }}=0,000$; MannWhitney-U-Test (gemütlich/ungemütlich*25 und jünger/66 und älter): $z=-4,226, p_{\text {zweiseitig }}=0,000$; Mann-WhitneyU-Test (vertraut/distanziert*25 und jünger/66 und älter): $z=-3,233, p_{\text {zweiseitig }}=0,001$; Mann-Whitney-U-Test (freundlich/unfreundlich*25 und jünger/66 und älter): $z=-5,448, p_{\text {zweiseitig }}=0,000$.

52 Spearman-Korrelation (modern/altmodisch*Altersgruppen): $r_{s}=-0,106, d f=530, p_{\text {zweiseitig }}=0,014$; SpearmanKorrelation (cool/uncool*Altersgruppen): $r_{s}=-0,233, d f=530, p_{\text {zweiseitig }}=0,000$.

53 Kruskal-Wallis-H-Test (modern/altmodisch*Altersgruppen): $\chi^{2}=7,651, d f=3, p_{\text {zweiseitig }}=0,54$; Kruskal-WallisH-Test (cool/uncool*Altersgruppen): $\chi 2=32,898, d f=3, p_{\text {zweiseitig }}=0,000$; Mann-Whitney-U-Test (cool/uncool*25 und jünger/66 und älter): $z=-3,695, p_{\text {zweiseitig }}=0,000$. 
Zur Bewertung von Niederdeutsch und lokalem Substandard in Hamburg

Befragtengruppe am negativsten beurteilt wird. Es kristallisiert sich heraus, dass die Hamburger Umgangssprache für jüngere Hamburger augenscheinlich ein hohes Prestige besitzt, während das Niederdeutsche weniger prestigebehaftet zu sein scheint. Dieser Hypothese ist im Zuge weiterer Analysen zur quantitativen Teilstudie nachzugehen.

\subsection{Zur Korrelation von Herkunftsregion und Bewertung der hamburgischen Um- gangssprache}

Beim Faktor „Herkunft“ ist die Nähe des Geburtsortes zu Hamburg beim Substandard wie beim Niederdeutschen mit positiveren Bewertungen verbunden (Tabelle 25). Dies betrifft vor allem die Eigenschaften ,(eher) vertraut“, „,(eher) freundlich“ und ,(eher) geradeheraus“.

\begin{tabular}{|l|l|l|l|l|}
\hline \multicolumn{1}{|c|}{ Herkunft } & $\begin{array}{l}\text { Hamburg } \\
\text { in \% }\end{array}$ & $\begin{array}{l}\text { Nord- } \\
\text { deutschland } \\
\text { in \% }\end{array}$ & $\begin{array}{l}\text { Mittel-/Süd- } \\
\text { deutschland } \\
\text { in \% }\end{array}$ & $\begin{array}{l}\text { Ausland } \\
\text { in \% }\end{array}$ \\
\hline (eher) vertraut & $78,9(236)$ & $65,9(89)$ & $57,4(39)$ & $57,2(16)$ \\
\hline $\begin{array}{l}\text { (eher) freund- } \\
\text { lich }\end{array}$ & $73,7(224)$ & $71,5(95)$ & $65,2(45)$ & $46,7(14)$ \\
\hline $\begin{array}{l}\text { (eher) gerade- } \\
\text { heraus }\end{array}$ & $88,5(269)$ & $86,2(115)$ & $80,8(55)$ & $80,0(24)$ \\
\hline (eher) modern & $9,8(29)$ & $14,5(19)$ & $14,7(10)$ & $17,2(5)$ \\
\hline (eher) grob & $49,8(146)$ & $50,7(66)$ & $41,8(28)$ & $41,3(12)$ \\
\hline (eher) cool & $33,4(98)$ & $41,2(54)$ & $32,3(22)$ & $30,0(9)$ \\
\hline
\end{tabular}

Tabelle 25: Korrelation von Herkunft und Bewertung; Tendenz positiv vs. Tendenz negativ

Zwischen der Herkunftsregion sowie den Merkmalspaaren „,vertraut - distanziert“, „freundlich - unfreundlich“ und ,geradeheraus - umständlich“ können signifikante positive Rangkorrelationen eruiert werden. ${ }^{54}$ Zugleich kommt die verteilungsfreie Varianzanalyse zu dem Ergebnis, dass diese Bewertungen hinsichtlich ihrer zentralen Tendenzen voneinander abweichen. ${ }^{55}$ Nach Adjustierung des Alphaniveaus stellen sich die verschiedenen Eigenschaftszuschreibungen in den Gruppen der im Ausland bzw. in Mittel-/Süddeutschland Geborenen gegenüber denjenigen, die in Hamburg geboren sind, als überzufällig heraus. ${ }^{56}$ Im Hinblick auf das Eigenschaftspaar ,,vertraut - distanziert“ kann darüber hinaus die Bewertungstendenz der aus Norddeutschland und der aus Hamburg stammenden Gewährspersonen als differierend

\footnotetext{
54 Spearman-Korrelation (vertraut/distanziert*Herkunftsregion): $r_{s}=0,253, d f=528, p_{\text {zweiseitig }}=0,000$; SpearmanKorrelation (freundlich/unfreundlich*Herkunftsregion): $r_{s}=0,133, d f=534, p_{\text {zweiseitig }}=0,002$; Spearman-Korrelation (geradeheraus/umständlich*Herkunftsregion): $r_{s}=0,133, d f=533, p_{\text {zweiseitig }}=0,002$.

55 Kruskal-Wallis-H-Test (vertraut/distanziert*Herkunftsregion): $\chi^{2}=34,709, d f=3, p_{\text {zweiseitig }}=0,000$; KruskalWallis-H-Test (freundlich/unfreundlich*Herkunftsregion): $\chi^{2}=9,856, d f=3, p_{\text {zweiseitig }}=0,020$; Kruskal-Wallis-HTest (geradeheraus/umständlich*Herkunftsregion): $\chi^{2}=11,218, d f=3, p_{\text {zweiseitig }}=0,011$.

56 Mann-Whitney-U-Test (vertraut/distanziert*Ausland/Hamburg): $z=-3,361, p_{\text {zweiseitig }}=0,001$; Mann-Whitney-UTest (vertraut/distanziert*Mittel- und Süddeutschland/Hamburg): $z=-4,993, p_{\text {zweiseitig }}=0,000$; Mann-Whitney-UTest (freundlich/unfreundlich*Ausland/Hamburg): $z=-2,083, p_{\text {zweiseitig }}=0,037$; Mann-Whitney-U-Test (freundlich/unfreundlich*Mittel- und Süddeutschland/Hamburg): $z=-2,436, \quad p_{\text {zweiseitig }}=0,015 ;$ Mann-Whitney-U-Test (geradeheraus/umständlich*Ausland/Hamburg): $z=-2,186, p_{\text {zweiseitig }}=0,029 ;$ Mann-Whitney-U-Test (geradeheraus/umständlich*Mittel- und Süddeutschland/Hamburg): $z=-2,826, p_{\text {zweiseitig }}=0,005$.
} 
erachtet werden. ${ }^{57}$ In Bezug auf die Pole „modern - altmodisch“, „,vornehm - grob“ und „,cool - uncool“ können hingegen keine signifikanten Korrelationen zur Herkunftsregion festgestellt werden. ${ }^{58}$ Beobachtete Unterschiede in den relativen Häufigkeiten wie z. B., dass die Gewährspersonen aus entfernteren Geburtsorten häufiger die Option ,(eher) modern“ wählen, können durch die statistische Auswertung nicht als signifikant verifiziert werden. Vielmehr weisen die Bewertungen der untersuchten Stichproben dieselbe Tendenz auf. ${ }^{59}$ Insgesamt zeigt der hamburgische Substandard unter dem potentiellen Einflussfaktor „Herkunftsregion“ dieselben Trends wie das Niederdeutsche. Lediglich für die Eigenschaft „(eher) geradeaus“ ist zu konstatieren, dass die Befragten, die im Ausland geboren sind, dieses Merkmal für die hamburgische Umgangssprache häufiger nennen als für das Niederdeutsche.

\subsection{Zwischenergebnis: Faktoren für die Bewertung der hamburgischen Umgangs- sprache}

Ebenso wie bei den Daten zum Niederdeutschen ein Zusammenhang zwischen positiver Bewertung und Sprachkompetenz festgestellt werden konnte, ist für die hamburgische Umgangssprache ein Zusammenhang zwischen Gebrauch und Bewertung zu erkennen. Diejenigen, die selbst regionale Merkmale in ihrer Sprache verwenden, beurteilen die regionale Sprachform tendenziell positiver als andere. In der Regel ist eine positivere Bewertung insbesondere hinsichtlich der emotiven Eigenschaften wie beim Niederdeutschen auch an ein höheres Alter gebunden. Ein sichtbarer Unterschied wird jedoch für die Eigenschaften „,cool“ und „modern“ deutlich. Die jüngste Befragtengruppe beurteilt die hamburgische Umgangssprache deutlich mehr als „,cool“ und etwas weniger hervorstechend auch stärker als „,modern“ als die älteste Gruppe. Zugleich fällt bei der jüngsten Gruppe die Beurteilung der Umgangssprache positiver aus als die des Niederdeutschen. Hinsichtlich der Herkunftsregion der Befragten zeigen sich beim Niederdeutschen und bei der hamburgischen Umgangssprache größtenteils dieselben Tendenzen, allerdings korreliert eine größere Distanz des Geburtsortes zu Hamburg mit einer häufigeren Einschätzung der hamburgischen Umgangssprache als „modern“.

\section{$6 \quad$ Ein kurzes Fazit}

Die Analyse bestätigt die bereits aus anderen Studien bekannte Tendenz einer insgesamt positiven Beurteilung des Niederdeutschen. Die Stichprobenvergleiche nach den Faktoren Kompetenz, Alter, Geschlecht, Bildung und Herkunftsregion unterscheiden sich vor allem hinsichtlich ihres Ausprägungsgrads der zwei möglichen Bewertungsstufen eines Merkmals, wobei die Grundtendenz der Zuschreibungen dieselbe bleibt. Es ist daher nicht verwunderlich, dass die ermittelten Zusammenhänge zwischen den zugrunde gelegten Variablen und den Sprachbewertungen ausnahmslos schwach bis mittelstark ausfallen. Vielmehr indizieren die Ergebnisse, dass die Eigenschaftszuschreibungen zwar bis zu einem gewissen Grad mit den

\footnotetext{
${ }^{57}$ Mann-Whitney-U-Test (vertraut/distanziert*Norddeutschland/Hamburg): $z=-3,195, p_{\text {zweiseitig }}=0,001$.

58 Spearman-Korrelation (modern/altmodisch*Herkunftsregion): $r_{s}=-0,004, d f=522, p_{\text {zweiseitig }}=0,923$; SpearmanKorrelation (vornehm/grob*Herkunftsregion): $\quad r_{s}=-0,045, \quad d f=517, \quad p_{\text {zweisetig }}=0,302 ; \quad$ Spearman-Korrelation (cool/uncool*Herkunftsregion): $r_{s}=0,011, d f=521, p_{\text {zweiseitig }}=0,798$.

59 Kruskal-Wallis-H-Test (modern/altmodisch*Herkunftsregion): $\chi^{2}=0,160, d f=3, p_{\text {zweiseitig }}=0,984$; KruskalWallis-H-Test (vornehm/grob*Herkunftsregion): $\quad \chi^{2}=2,308, \quad d f=3, \quad p_{\text {zweiseitig }}=0,511 ; \quad$ Kruskal-Wallis-H-Test (cool/uncool*Herkunftsregion): $\chi^{2}=3,602, d f=3, p_{\text {zweiseitig }}=0,308$.
} 
Zur Bewertung von Niederdeutsch und lokalem Substandard in Hamburg

untersuchten Faktoren im Zusammenhang stehen, das allgemeine stereotype Bild des Niederdeutschen (als humorvolle, gemütliche und sympathische Nahsprache) bleibt jedoch unberührt. In der Gesamtbewertung stehen insbesondere die emotiven Eigenschaften im Mittelpunkt, stilistische Zuschreibungen werden zurückhaltender vorgenommen. Dabei wird das Niederdeutsche von kompetenten und älteren Sprechern, von Personen, die aus dem niederdeutschen Sprachraum stammen, und solchen, die überwiegend ein eher niedriges Bildungsniveau aufweisen, positiver wahrgenommen. Geschlechtsspezifische Unterschiede können dagegen nur für einzelne Merkmalspaare festgehalten werden, wobei die männlichen Befragten zu positiveren Bewertungen tendieren. Damit können die eingangs formulierten Hypothesen weitestgehend bestätigt werden. Lediglich das von Möller beschriebene „Imageproblem“ (Möller 2008: 74) des Niederdeutschen bei Personen mit niedrigem Bildungsabschluss schlägt sich in den Ergebnissen der NiH-Befragung nicht nieder. Unerwartet ist darüber hinaus das Ergebnis, dass Niederdeutsch und hamburgischer Substandard gleichartige Trends zeigen. Es treten dieselben Bewertungstendenzen bei den emotiven Eigenschaften hervor, wobei für die einzelnen Eigenschaften teilweise Unterschiede in der Rangfolge bestehen. Analog zum Niederdeutschen korrelieren auch bei der hamburgischen Umgangssprache höheres Alter und aktiver Sprachgebrauch mit positiver Einstellung. Unterschiede werden bei einzelnen Befragtengruppen deutlich, so die frequentere Bewertung des hamburgischen Substandards der jüngsten Gruppe als „,cool“ und „,modern“. Die Analysen der Bewertung sind künftig vor allem auf die Frage nach dem Zusammenhang mit der Ortseinstellung auszudehnen, um das Identifikationspotential des Niederdeutschen und des hamburgischen Substandards zu überprüfen. Zudem wäre es reizvoll, die Zuschreibungen zu den lokalen Sprachformen Hamburgs mit denen anderer Städte zu vergleichen, z. B. Berlin, Köln, München, Wien, Zürich oder Luxemburg.

\section{Literaturverzeichnis}

Bichel, Ulf (1985): „Die Überlagerung des Niederdeutschen durch das Hochdeutsche“. In: Besch, Werner/Reichmann, Oskar/Sonderegger, Stefan (eds.): Sprachgeschichte. Ein Handbuch zur Geschichte der deutschen Sprache und ihrer Erforschung. 2. Halbbd. Berlin/New York, de Gruyter: 1865-1873. (= Handbücher zur Sprach- und Kommunikationswissenschaft 2.2).

Cuonz, Christina (2014): Sprachliche Werturteile von Laien. Eine sozio-kognitive Analyse. Tübingen: Francke. (= Basler Studien zur deutschen Sprache und Literatur 93).

Gärtig, Anne-Kathrin/Plewnia, Albrecht/Rothe, Astrid (2010): Wie Menschen in Deutschland über Sprache denken. Ergebnisse einer bundesweiten Repräsentativerhebung zu aktuellen Spracheinstellungen. Mannheim: Institut für Deutsche Sprache. (= Arbeitspapiere und Materialien zur deutschen Sprache 40).

Gries, Stefan Thomas (2008): Statistik für Sprachwissenschaftler. Göttingen: Vandenhoeck \& Ruprecht.

Hettler, Yvonne (2013): „,Nach Müllers geht man nicht!‘ Zur Salienz und Bewertung morpho-syntaktischer Phänomene in Bremen und Hamburg“. In: Hettler, Yvonne/Jürgens, Carolin/Langhanke, Robert/Purschke, Christoph (eds.): Variation, Wandel, Wissen. Studien zum Hochdeutschen und Niederdeutschen. Frankfurt a. M., Lang: 161-183. (=Sprache in der Gesellschaft 32). 
Hettler, Yvonne (2014): „Salienz, Bewertung und Realisierung regionaler Merkmale in Norddeutschland“. Linguistik online 66: 71-90.

Hundt, Markus (1992): Einstellungen gegenüber dialektal gefärbter Standardsprache. Eine empirische Untersuchung zum Bairischen, Hamburgischen, Pfälzischen und Schwäbischen. Stuttgart: Steiner. (=Zeitschrift für Dialektologie und Linguistik, Beihefte 78).

Hundt, Markus/Anders, Christina Ada/Lasch, Alexander (eds.) (2010): "perceptual dialectology”. Neue Wege der Dialektologie. Tagungsband mit Beiträgen der Internationalen Fachtagung zur Wahrnehmungsdialektologie an der Christian-Albrechts-Universität zu Kiel (22.-24. Mai 2008). Berlin/New York: de Gruyter. (=Linguistik-Impulse \& Tendenzen 38).

Ipsos (2007): Unveröffentlichtes Tabellenmaterial der Umfrage des Ipsos-Instituts im Auftrag des Instituts für niederdeutsche Sprache 2007; Ergebnisse publiziert in: Möller, Frerk (2008): Plattdeutsch im 21. Jahrhundert. Bestandsaufnahme und Perspektiven. Leer: Schuster. (= Schriften des Instituts für Niederdeutsche Sprache 34).

Jürgens, Carolin (2015): Niederdeutsch im Wandel. Sprachgebrauchswandel und Sprachwahrnehmung in Hamburg. Hildesheim: Olms. (= Deutsche Dialektgeographie 119).

Jürgens, Carolin (2016): „Regionale Identität per Einkaufstüte. Eine Fallstudie zum Enregisterment des Niederdeutschen in Hamburg“. In: Bieberstedt, Andreas/Ruge, Jürgen/Schröder, Ingrid (eds.): Hamburgisch. Struktur, Gebrauch, Wahrnehmung der Regionalsprache im urbanen Raum. Frankfurt a. M., Lang: 307-343. (=Sprache in der Gesellschaft 34).

Jürgens, Carolin/Schröder, Ingrid (2016): „Sprachstereotype und ihre Realisierungen im Gespräch am Beispiel des Niederdeutschen“. In: Bieberstedt, Andreas/Ruge, Jürgen/Schröder, Ingrid (eds.): Hamburgisch. Struktur, Gebrauch, Wahrnehmung der Regionalsprache im urbanen Raum. Frankfurt a. M., Lang: 345-385. (= Sprache in der Gesellschaft 34).

Lasagabaster, David (2004): „Attitude/Einstellung“. In: Ammon, Ulrich/Dittmar, Norbert/Mattheier, Klaus J./Trudgill, Peter (eds.): Sociolinguistics/Soziolinguistik. An International Handbook of the Science of Language and Society/Ein internationales Handbuch zur Wissenschaft von Sprache und Gesellschaft. 1. Teilbd. 2., vollständig neu bearb. und erw. Auflage. Berlin/New York, de Gruyter: 399-405. (=Handbücher zur Sprachund Kommunikationswissenschaft 3.1).

Lesle, Ulf-Thomas (1986): Das niederdeutsche Theater. Von „, völkischer Not“ zum Literaturtrost. Hamburg: Christians.

Möhn, Dieter (2003): „Die Stadt in der neueren deutschen Sprachgeschichte I: Hamburg“. In: Besch, Werner/Betten, Anne/Reichmann, Oskar/Sonderegger, Stefan (eds.): Sprachgeschichte. Ein Handbuch zur Geschichte der deutschen Sprache und ihrer Erforschung. 3. Teilbd. 2., vollst. neu bearb. und erw. Auflage. Berlin/New York, de Gruyter: 2297-2312. (= Handbücher zur Sprach- und Kommunikationswissenschaft 2.3).

Möller, Frerk (2008): Plattdeutsch im 21. Jahrhundert. Bestandsaufnahme und Perspektiven. Leer: Schuster. (= Schriften des Instituts für Niederdeutsche Sprache 34).

Möller, Frerk (2010): „Platt in Hamburg anno 2007“. In: Müns, Wolfgang (ed.): Man mag sik kehrn un kanten, as man will, noch jümmer is der'n Eck, wo man ni wen is. 100. Jahrgang der Zeitschrift Quickborn. Festschrift. Hamburg, Quickborn-Verlag: 549-565. (= Quickborn-Bücher 100/101). 
Zur Bewertung von Niederdeutsch und lokalem Substandard in Hamburg

Niebaum, Hermann/Macha, Jürgen (2014): Einführung in die Dialektologie des Deutschen. 3. Auflage. Berlin/Boston: de Gruyter. (= Germanistische Arbeitshefte 37).

Reershemius, Gertrud (2004): Niederdeutsch in Ostfriesland. Zwischen Sprachkontakt, Sprachveränderung und Sprachwechsel. Stuttgart: Steiner. (= Zeitschrift für Dialektologie und Linguistik, Beihefte 119).

Schoel, Christiane/Stahlberg, Dagmar (2012): „Spracheinstellungen aus sozialpsychologischer Perspektive II: Dialekte“. In: Eichinger, Ludwig Maximilian/Plewnia, Albrecht/Schoel, Christiane/Stahlberg, Dagmar (eds.): Sprache und Einstellungen. Spracheinstellungen aus sprachwissenschaftlicher und sozialpsychologischer Perspektive. Tübingen, Narr: 205-225. (= Studien zur deutschen Sprache 61).

Schröder, Ingrid (2007): „Stigmatisierung durch Sprachwahl: Niederdeutsch in den Moralischen Wochenschriften des 18. Jahrhunderts“. In: Hickethier, Knut/Schumann, Katja (eds.): Die schönen und die nützlichen Künste. Literatur, Technik und Medien seit der Aufklärung. Harro Segeberg zum 65. Geburtstag. München/Paderborn, Fink: 83-93.

Schröder, Ingrid (2010): „Plattdeutsch in Hamburg. Sprachwahl als Mittel zur Konstruktion lokaler Identität?“ In: Müns, Wolfgang (ed.): Man mag sik kehrn un kanten, as man will, noch jümmer is der'n Eck, wo man ni wen is. 100. Jahrgang der Zeitschrift „, Quickborn “. Festschrift. Hamburg, Quickborn-Verlag: 585-601. (= Quickborn-Bücher 100/101).

Schröder, Ingrid (2012): „De Hamborger Jung. Zur Gestaltung eines Stereotyps zu Beginn des 20. Jahrhunderts“. In: Hempel, Dirk/Schröder, Ingrid (eds.): Andocken. Hamburgs Kulturgeschichte 1848-1933. Hamburg, DOBU-Verlag: 473-486.

Schröder, Ingrid (2013): „Sprache, Stadt, Stereotyp. Zur sozialsymbolischen Funktion des Niederdeutschen im urbanen Raum“. In: Durrell, Martin (ed.): Vielheit und Einheit der Germanistik weltweit. Bd. 17: Diachronische, diatopische und typologische Aspekte des Sprachwandels. Frankfurt a. M., Lang: 377-382. (= Publikationen der Internationalen Vereinigung für Germanistik (IVG) 17).

Schröder, Ingrid (2015): „,Von der Dialektologie zur Regionalsprachenforschung - eine norddeutsche Perspektivierung“. In: Elmentaler, Michael/Hundt, Markus/Schmidt, Jürgen Erich (eds.): Deutsche Dialekte. Konzepte, Probleme, Handlungsfelder. Akten des 4. Kongresses der Internationalen Gesellschaft für Dialektologie des Deutschen. Stuttgart, Steiner: 2557. (=Zeitschrift für Dialektologie und Linguistik, Beihefte 158).

Schröder, Ingrid (i. E.): „Sprachwechsel in Hamburg. Vom Niederdeutschen zum Hochdeutschen“. In: Béhar, Roland/Blanco, Mercedes/Hafner, Jochen (eds.): Villes à la croisée des langues (XVIe-XVIIe siècles). Palerme, Naples, Milan, Anvers et Hambourg. Travail fondé sur les résultats de l'atelier $n^{\circ} 5$ EUROLAB. Genf: Droz.

Sinus-Institut (2015): Informationen zu den Sinus-Milieus 2015/16. Stand: 09/2015. www.sinus-institut.de/veroeffentlichungen/downloads/download/informationen-zu-densinus-milieusR/download-file/1075/download-a/download/download-c/Category/. [08.08.2016].

Stellmacher, Dieter (1987): Wer spricht Platt? Zur Lage des Niederdeutschen heute. Eine kurzgefaßte Bestandsaufnahme. Leer: Schuster. (= Schriften des Instituts für Niederdeutsche Sprache 14).

Stellmacher, Dieter (1995): Niedersächsischer Dialektzensus. Statistisches zum Sprachgebrauch im Bundesland Niedersachsen. Stuttgart: Steiner. (=Zeitschrift für Dialektologie und Linguistik, Beihefte 88). 